\title{
A new study of associating inhomogeneous fluids with classical density functional theory
}

Camacho Vergara, Edgar L.; Kontogeorgis, Georgios M.; Liang, Xiaodong

Published in:

Molecular Physics

Link to article, DOI:

10.1080/00268976.2020.1725668

Publication date:

2020

Document Version

Peer reviewed version

Link back to DTU Orbit

Citation (APA):

Camacho Vergara, E. L., Kontogeorgis, G. M., \& Liang, X. (2020). A new study of associating inhomogeneous fluids with classical density functional theory. Molecular Physics, 118(9-10), [ e1725668 ].

https://doi.org/10.1080/00268976.2020.1725668

\section{General rights}

Copyright and moral rights for the publications made accessible in the public portal are retained by the authors and/or other copyright owners and it is a condition of accessing publications that users recognise and abide by the legal requirements associated with these rights.

- Users may download and print one copy of any publication from the public portal for the purpose of private study or research.

- You may not further distribute the material or use it for any profit-making activity or commercial gain

- You may freely distribute the URL identifying the publication in the public portal

If you believe that this document breaches copyright please contact us providing details, and we will remove access to the work immediately and investigate your claim. 


\title{
A new study of associating inhomogeneous fluids with classical density functional theory
}

\author{
Edgar L. Camacho Vergara ${ }^{a}$, Georgios M. Kontogeorgis ${ }^{\mathrm{a}}$ and Xiaodong Liang ${ }^{\mathrm{a}}$ \\ ${ }^{a}$ CERE - Center for Energy Resources Engineering, Department of Chemical and Biochemical \\ Engineering at the Technical University of Denmark, 2800 Kgs. Lyngby, Denmark
}

\section{ARTICLE HISTORY}

Compiled January 30, 2020

\begin{abstract}
A new density functional for the study of associating inhomogeneous fluids based on Wertheim's first-order thermodynamic perturbation theory is presented and compared to the most currently used associating density functionals. This functional is developed using the weighted density approximation (WDA) in the range of association of hard spheres. We implement this functional within the framework of classical density functional theory together with modified fundamental measure theory to account for volume exclusion of hard spheres. This approach is tested against molecular simulations from literature of pure associating hard spheres and mixtures of non-associationg and associating hard spheres with different number of bonding sites close to a hard uniform wall. Furthermore, we compare and review our results with the performance of associating functionals from literature, one based on fundamental measure theory (aFMT) and the inhomogeneous version of Wertheim's perturbation theory (iSAFT). Results obtained with classical DFT and the three functionals show excellent agreement with molecular simulations in systems with one hard wall. For the cases of small pores where only one or two layers of fluid are allowed discrepancies between results with classical DFT and molecular simulations were found.
\end{abstract}

\section{KEYWORDS}

Classical density functional theory; confined fluids; associating fluids

\section{Introduction}

The study of inhomogeneous fluids has great relevance for both theory and industries as it involves a wide range of applications. An inhomogeneous fluid can be understood as a system containing one or more fluids that are under the effect of an external potential which causes local variation in the microscopic density distribution of the particles [1]. The shape of the potential exerted to the system of interest affects the structure of the fluids and diverts it from the homogeneous or uniform behavior of bulk fluids. Such systems can be found close to surfaces or interfaces where the uniformity of the particle density is broken, which results in properties like interfacial tension, when an interface between two fluid phases is present, or adsorption, when a fluid phase is in contact with a solid. In order to successfully understand the underlying nature of 
inhomogeneous fluids it is necessary to develop thermodynamic models that allow an accurate description of the inter- and intra-particle interactions and additionally with the external potential.

The most used methods in the study inhomogeneous fluids are molecular simulations, classical density functional theory (DFT) and integral-equation theories [2]. For the latter we refer to Ref. 1. From certain perspective, molecular simulations are the most promising methods as they allow a very detailed description of the molecules, interactions and system geometries. However, even with the ever increasing computer power nowadays molecular simulations require a substantial amount of computational resources and post-processing of the results. On the other hand, classical DFT combines the versatility of bulk thermodynamic models with rigorous concepts of statistical mechanics to provide a suitable framework for the study of inhomogeneous fluids that only require a fraction of the calculation time required by molecular simulations, without compromising accuracy in the results. That is why one of the first steps in the development of any classical DFT model, researches focus their attention in comparing the performance between these two approaches. Currently, results obtained with classical DFT have shown to be in excellent agreement with molecular simulations in the determination of the equilibrium particle density of confined simple fluids [3-10]. We refer to Refs. 2, 11, 12 for a more comprehensive review and examples of classical DFT.

The key concept in classical DFT is the definition of an adequate Helmholtz free energy density functional that accurately describes the interaction potential of the particles in the non-uniform fluid. Generally, the free energy functional of the inhomogeneous fluid is approximated from its bulk counterpart defined by a thermodynamic equation of state. Wertheim's thermodynamic perturbation theory (TPT) [13-16] establishes a solid basis where many researchers develop their classical DFT implementations from, which enables the consideration of different contributions to the Helmholtz free energy functional. These contributions depend on the nature of the particle interactions and are considered perturbations from a reference state. One of the main examples in the development of thermodynamic models for bulk fluids based on TPT is the statistical association fluid theory (SAFT) [17-19] and its variants [20-22]. What most SAFT versions have in common is the use of the hard sphere model as the reference state, and the effects of chain formation, dispersion forces and association between particles are added as perturbation. In a similar way, a classical DFT Helmholtz free energy functional commonly uses the hard sphere model as reference in the form of the fundamental measure theory (FMT) originally proposed by Rosenfeld [23] and later modified simultaneously by $\mathrm{Yu}$ and $\mathrm{Wu}$ [5], and Roth et al. [3] Modified FMT has proven to be an excellent choice as it accurately describes the density distribution of hard spheres in confined geometries in comparison with molecular simulations. $[4,5]$ From this starting point, different functionals and techniques have been established to take into account additional contributions to the free energy which have lead to the creation of different versions of classical DFT implementations in a similar manner as there are different variants of SAFT. Some specific classical DFT examples include SAFT-VR [24], iSAFT [8, 10], PC-SAFT [25, 26] and the work of Wu et al. [6, 7, 27]. It is relevant to point out that in the bulk limit, where the particle density distribution tends to the bulk density $\rho(\mathbf{r}) \rightarrow \rho^{\text {bulk }}$, the inhomogeneous functionals reduce to the bulk expression of the free energy density.

Particle interactions due to association are strongly related to fluids that are able to form hydrogen bonding, e.g. water, alcohols and acids. For this kind of fluids, association between molecules play an important role in their thermodynamic properties 
as they heavily contribute to the particle interaction potential [19]. One theory for the study of bulk associating fluids that may be considered the most successful is the one of Wertheim's $[13,14]$, as it has been extensively used in the study of bulk fluids with one, two or four associating sites [17, 21, 22, 28]. From the side of the inhomogeneous case, the works of Segura et al. [29-32] introduced a strong foundation by obtaining results of confined associating fluids with molecular simulations and classical DFT. Their classical DFT implementation accounted for volume exclusion of hard spheres with help of a density dependent weighted density labeled Tarazona Mark II [33]. In order to account for association Segura et al. implemented two functionals, (1) one using the inhomogeneous form of Wertheim's TPT and (2) the sencond using the same density dependent weighted density as defined in Mark II. They tested their implementation against molecular simulations of confined associating fluids with four bonding sites and concluded that the weighted density approach (2) was superior in terms of agreement with simulations, and required considerable less computational time [30]. Following works implemented the same weighted density functional to study confinement of associating hard spheres with one and two association sites,[31] and extended it for binary mixtures between hard spheres and an associating fluid with four sites [32]. In most of the cases studied good agreement between simulations and classical DFT was found, with only a few exceptions where the packing fraction is large and the systems are mainly dominated by collision of the particles.

The shortcoming found in Segura's classical DFT is mainly attributed to the functional used for hard spheres (Mark II), point that was noted by $\mathrm{Yu}$ and $\mathrm{Wu}$ [6] when they used the modified FMT to account for volume exclusion and introduced a new association functional built with Wertheim's TPT and weighted densities defined in FMT. The association functional developed by $\mathrm{Yu}$ and $\mathrm{Wu}$ will be labeled aFMT from here on. The introduction of modified FMT considerably improved the performance of classical DFT in these cases of large packing fractions, and together with their new association functional excellent agreement was found when compared to molecular simulations of confined hard spheres with four association sites and also in mixtures with non-associating spheres. More recently, Bymaster and Chapman [10] took on the work of Segura et al. [30] and improved their association functional based on the inhomogeneous form of Wertheim's TPT (1) with slight modifications in the way the cavity correlation function of the inhomogeneous fluid is approximated. Bymaster and Chapman showed that their association functional, labeled iSAFT, had an excellent agreement with simulations of associating fluids and superior than the original work of Segura et al. [30].

Nowadays, the functionals aFMT and iSAFT are the most used by researchers interested in the study of inhomogeneous associating fluids. They both offer excellent accuracy when compared with simulations but there has not been any thorough comparison between the two that we know of. $\mathrm{Yu}$ and $\mathrm{Wu}$ [6] used modified FMT to account for volume exclusion, whereas Bymaster and Chapman [10] used the original FMT [23]. In some cases, iSAFT shows better agreement with simulations for dimerizing spheres.[10] However, this comes with a cost as the computational time required by iSAFT is larger when compared to aFMT giving the nature of the calculation of the fraction of non-bonded species $[6,10]$. As mentioned before, for the study of more complex fluids, additional contributions to the Helmholtz free energy must be taken into account in order to obtain an accurate representation of their thermodynamic properties in inhomogeneous systems. Examples of these applications with iSAFT includes the study of interfaces with polymers, copolymers, surfactants, tethered polymers, among others [12]. Additionally, special attention has been given to the study of the 
phase behavior and properties of water in homogeneous systems. Hughes et al. [34] studied the structure of water around hydrophobic rods and the hydration energy of hard sphere solutes with aFMT, finding good agreement with molecular simulations of the same systems. More recently, Trejos et al. [35, 36] carried out studies of the phase behavior of water confined in attractive slit-like pores, where they were able to calculate the shift in the vapor-liquid envelope due to confinement for different pore sizes, and also the solvation force of the pore systems where they found excellent agreement with molecular simulation of water with different force fields.

The main focus of this work is the development of a density functional for associating fluids based on the weighted density approximation (WDA) [37, 38]. This WDA is a simple but capable approximation that consists in approximating the free energy functional by means of an averaged density and the free energy density of the bulk fluid. The averaged density is calculated with a weighting function that relates to an effective volume that depends on the range of interaction of the given contribution to the free energy. With this new implementation we hope to fill in the blanks where aFMT may fall short when compared with iSAFT but maintaining the computation time similar to aFMT. As secondary objective we carry out a thorough comparison of iSAFT and aFMT together with our new implementation against molecular simulations [30-32, 39] of associating hard spheres and mixtures with non-associating spheres close to an uniform hard wall. For this, we adopt modified FMT for volume exclusion for all our calculations and only consider association with the three functionals aforementioned. To model the different number of bonding sites, we appeal for the association schemes defined by Huang and Radosz [40, 41] for dimerizing spheres (1A), polymerizing spheres (2B) and a water-like model with four association sites $(4 \mathrm{C})$. The fraction of non-bonded monomers as a function of the distance from the wall is also compared with molecular simulations.

In the following, we first illustrate the models and methods used in this work in Section 2. In Section 2.1 we introduce some of the concepts of classical DFT and the Helmholtz free energy functional. Later on Section 2.2, we present the three association functionals, similarities and differences. In Section 3 the results from simulations and classical DFT with the three different functionals are compared for associating fluids confined in slit like pores. Here we systematically show results for the equilibrium density distribution of particles and fraction of monomers for schemes $1 \mathrm{~A}, 2 \mathrm{~B}$ and $4 \mathrm{C}$. Results for binary mixtures of hard non-associating spheres and associating spheres with four bonding sites can be found in Section 3.5. Finally, we conclude our findings and comparisons in Section 4.

\section{Models and method}

In the present work we consider the general case of a multicomponent model of associating and non-associating particles confined in a symmetrical slit-like pore composed of two uniform hard walls. For two given particles ( 1 and 2$)$ the interactions are described through a pairwise potential considering volume exclusion and association,

$$
u\left(\mathbf{r}_{12}, \boldsymbol{\omega}_{1}, \boldsymbol{\omega}_{2}\right)=u^{\mathrm{hs}}\left(\mathbf{r}_{12}\right)+\sum_{A} \sum_{B} u_{A B}^{\text {assoc }}\left(\mathbf{r}_{12}, \boldsymbol{\omega}_{1}, \boldsymbol{\omega}_{2}\right)
$$

Where $u^{\text {hs }}$ is the hard sphere potential, $u^{\text {assoc }}$ represents the association potential, $\mathbf{r}_{12}$ is the vector connecting the centers of particles 1 and $2, \boldsymbol{\omega}_{1}$ and $\boldsymbol{\omega}_{2}$ are the orientation 


\section{8 $1 \mathrm{~A}$}

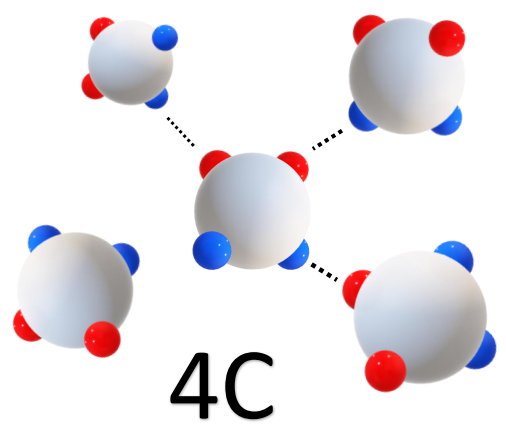

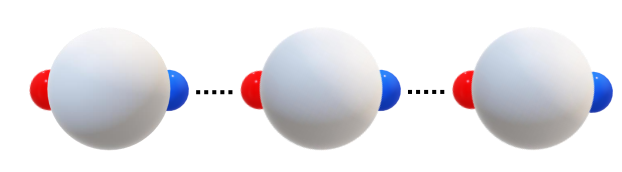

2B

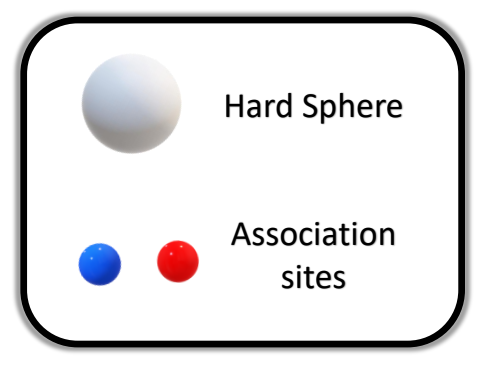

Figure 1. Depiction of the association schemes used in this work. (1A) dimerizing hard spheres with one association able to associate with itself; (2B) and (4C) have two and four association sites, respectively, and only association of different type of sites is allowed.

of the two particles, and the summation on the right is carried out over all association sites of the two particles. For two hard spheres of the same molecular diameter $\sigma$ the hard sphere potential is given by

$$
u^{\mathrm{hs}}\left(\mathbf{r}_{12}\right)= \begin{cases}\infty, & r_{12}<\sigma \\ 0, & r_{12} \geq \sigma\end{cases}
$$

Where $r_{12}$ is the distance from center to center of the two particles. The associating interactions $[6,10,30]$ between any two segments allowed to associate is

$$
u_{A B}^{\text {assoc }}\left(\mathbf{r}_{12}, \boldsymbol{\omega}_{1}, \boldsymbol{\omega}_{2}\right)= \begin{cases}-\varepsilon^{A B}, & r_{12}<r_{c}, \theta_{\mathrm{A} 1}<\theta_{c}, \theta_{\mathrm{B} 2}<\theta_{c} \\ 0, & \text { otherwise }\end{cases}
$$

where $\theta_{\mathrm{A} 1}$ and $\theta_{\mathrm{B} 2}$ are the angles between $\mathbf{r}_{12}$ and the vector connecting the center of each particle to its respective site $\mathrm{A}$ or $\mathrm{B}$, respectively. This implies that the sites are collocated in the particle in an off-center fashion. Depending on the scheme followed $[40,41]$, an associating particle has one (1A), two (2B) or four (4C) association sites as depicted in Fig. 1. For scheme 1A all association sites are considered the same and are allowed to associate with each other. For scheme $2 \mathrm{~B}$ each particle has two association sites labeled $A$ and $B$ situated at opposite sides of the particle and only $A B$ interactions are allowed, i.e. $\varepsilon_{A A}=\varepsilon_{B B}=0$. The scheme $4 \mathrm{C}$ consists of four association sites labeled $A, B, C$ and $D$ where only $A C, A D, B C$ and $B D$ are allowed to associate, i.e. $\varepsilon_{A A}=\varepsilon_{A B}=\varepsilon_{B B}=\varepsilon_{C C}=\varepsilon_{C D}=\varepsilon_{D D}=0$. Furthermore, it is assumed that all association energies are identical and that the limits of association are given by $r_{c}=1.05 \sigma$ and $\theta_{c}=27^{\circ}$. We used this values to be consistent in our 
comparison with molecular simulations and previous classical DFT implementations $[6,10,29-32]$.

As we are interested in the study of associating fluids under confinement in slit-like systems, it is required to define the external potential exerted by the hard walls to the fluid. This is accomplished by defining a wall potential as a function of the distance $z$ to the wall of the form

$$
V^{\operatorname{ext}}(z)= \begin{cases}0, & \sigma / 2<z<H-\sigma / 2 \\ \infty, & \text { otherwise }\end{cases}
$$

where $H$ is the effective size of the slit and $\sigma / 2$ represents the hard sphere radius.

\subsection{Classical Density Functional Theory}

The classical DFT framework was developed in the grand canonical ensemble, with fixed natural variables volume $V$, temperature $T$ and chemical potential of all components in the system $\mu_{i}$. The task for solving a classical DFT problem is of finding the equilibrium density profile of the inhomogeneous system by minimizing the grand potential functional of the density $\Omega[\boldsymbol{\rho}(\mathbf{r})]$, where $\boldsymbol{\rho}(\mathbf{r})=\left\{\rho_{1}(\mathbf{r}), \rho_{2}(\mathbf{r}), \ldots, \rho_{n}(\mathbf{r})\right\}$ is the vector density of all the components in the system. The grand potential is related to the Helmholtz free energy functional $F[\boldsymbol{\rho}(\mathbf{r})]$ according to

$$
\Omega[\boldsymbol{\rho}(\mathbf{r})]=F[\boldsymbol{\rho}(\mathbf{r})]+\sum_{i}^{n} \int d \mathbf{r} \rho_{i}(\mathbf{r})\left(V^{\operatorname{ext}}(z)-\mu_{i}\right)
$$

where $\rho_{i}(\mathbf{r})$ and $\mu_{i}$ are the local particle density and the chemical potential of species $i$ of the system. The equilibrium density profile $\boldsymbol{\rho}^{*}$ is found when the functional derivative of the grand potential with respect to the density is zero $\delta \Omega[\boldsymbol{\rho}(\mathbf{r})] / \delta \rho_{j}(\mathbf{r})=0$, and the following condition is satisfied

$$
\mu_{j}=\left.\frac{\delta F[\boldsymbol{\rho}(\mathbf{r})]}{\delta \rho_{j}(\mathbf{r})}\right|_{\boldsymbol{\rho}=\boldsymbol{\rho}^{*}}+V^{\mathrm{ext}}(z)
$$

The total Helmholtz free energy functional of the systems can be decomposed into an ideal part and an excess part $F[\boldsymbol{\rho}(\mathbf{r})]=F^{\mathrm{id}}[\boldsymbol{\rho}(\mathbf{r})]+F^{\mathrm{ex}}[\boldsymbol{\rho}(\mathbf{r})]$. The ideal part of the free energy functional is known exactly

$$
\beta F^{\mathrm{id}}[\boldsymbol{\rho}(\mathbf{r})]=\sum_{i}^{n} \int d \mathbf{r} \rho_{i}(\mathbf{r})\left(\ln \left(\rho_{i}(\mathbf{r}) \Lambda_{i}^{3}\right)-1\right)
$$

where $\Lambda$ is the thermal wavelength, and $\beta=\left(k_{B} T\right)^{-1}$ with $k_{B}$ as the Boltzmann constant. The value of $\Lambda$ is not relevant as it is density independent and can be considered a factor that does not affect the equilibrium conditions of the system. Following the basis of the perturbation approach, the excess part of the Helmholtz free energy functional can be approximated as a perturbation around a reference state. In this work the reference state is the hard sphere model which is perturbed by the 
associative contribution to the free energy as

$$
F^{\mathrm{ex}}[\boldsymbol{\rho}(\mathbf{r})]=F^{\mathrm{hs}}[\boldsymbol{\rho}(\mathbf{r})]+F^{\mathrm{as}}[\boldsymbol{\rho}(\mathbf{r})]
$$

The first term in the right hand side represents the hard sphere contribution, which can be evaluated with the modified fundamental measure theory developed simultaneously by $\mathrm{Yu}$ and $\mathrm{Wu}[5]$ and Roth et al. [3]. The hard sphere functional has the form of

$$
\beta F^{\mathrm{hs}}[\boldsymbol{\rho}(\mathbf{r})]=\int d \mathbf{r} f^{\mathrm{hs}}\left(\left\{n_{\alpha}(\mathbf{r})\right\}\right)
$$

Where $f^{\text {hs }}$ is the free energy density of the hard sphere, which is a function of four scalar and two vector weighted densities $n_{\alpha}(\mathbf{r})$. We refer to the work of $\mathrm{Yu}$ and $\mathrm{Wu}$ [5] for detailed description of these quantities.

\subsection{Association functionals}

We consider three association functionals, the one developed by $\mathrm{Yu}$ and $\mathrm{Wu}[6]$ based on the fundamental measure theory (aFMT), and the inhomogeneous association functional (iSAFT) developed by Bymaster and Chapman [10]. For the last, we propose a new implementation based on WDA that uses the free energy density of the associating bulk fluid. For the sake of clarity, we are going to introduce the main equations of these functionals.

In aFMT, the association functional is given by

$$
\beta F^{\mathrm{as}}[\boldsymbol{\rho}(\mathbf{r})]=\int d \mathbf{r} f^{\mathrm{as}}\left(\left\{n_{\alpha}(\mathbf{r})\right\}\right)
$$

Where similar to FMT, $f^{\text {as }}$ is a free energy density that is a function of some of the weighted densities defined in FMT $\alpha=0,2,3, V 2$, e.g. see equations (11)-(15) in Ref. 6. The free energy density is

$$
\begin{aligned}
f^{\mathrm{as}}\left(\left\{n_{\alpha}(\mathbf{r})\right\}\right)= & \sum_{i}^{n} n_{0 i}(\mathbf{r}) \zeta_{i}(\mathbf{r}) \times \\
& \sum_{A_{i}}^{\left\{A_{i}\right\}}\left(\ln \chi_{A_{i}}\left(\left\{n_{\alpha}(\mathbf{r})\right\}\right)-\frac{\chi_{A_{i}}\left(\left\{n_{\alpha}(\mathbf{r})\right\}\right)}{2}+\frac{1}{2}\right)
\end{aligned}
$$

Where $n_{0 i}$ and $\zeta_{i}$ are a weighted density and a correction factor for component $i$. Also appearing in Eq. (11) is the fraction of particles not bonded to site $A$ of component $i$ and the inner summation is carried out over all the association sites of the component. This fraction is calculated from

$$
\begin{aligned}
& \chi_{A_{i}}\left(\left\{n_{\alpha}(\mathbf{r})\right\}\right)= \\
& \left(1+\sum_{j}^{n} n_{0 j}(\mathbf{r}) \zeta_{j}(\mathbf{r}) \sum_{B_{j}}^{\left\{B_{j}\right\}} \chi_{B_{j}}\left(\left\{n_{\alpha}(\mathbf{r})\right\}\right) \Delta^{A_{i} B_{j}}\left(\left\{n_{\alpha}(\mathbf{r})\right\}\right)\right)^{-1},
\end{aligned}
$$


where

$$
\Delta^{A_{i} B_{j}}\left(\left\{n_{\alpha}(\mathbf{r})\right\}\right)=\kappa^{A_{i} B_{j}} f^{A_{i} B_{j}} g_{i j}^{\sigma \sigma}\left(\left\{n_{\alpha}(\mathbf{r})\right\}\right)
$$

is the association strength between two sites $A_{i}$ and $B_{j}, f^{A_{i} B_{j}}=\exp \left(\beta \varepsilon^{A_{i} B_{j}}\right)-1$ is the Mayer function [6], and $\varepsilon^{A_{i} B_{j}}$ is the association energy. The bonding volume between two particles is considered a constant[10, 30-32]

$$
\kappa^{A_{i} B_{j}}=K^{\mathrm{aFMT}}=\pi\left(1-\cos \theta_{c}\right)^{2} \sigma^{2}\left(r_{c}-\sigma\right) .
$$

Finally, $g_{i j}^{\sigma \sigma}\left(\left\{n_{\alpha}(\mathbf{r})\right\}\right)$ is the contact value of the bulk cavity correlation function adapted to modified FMT.[6]

The iSAFT functional is an improved version of the inhomogeneous form of Wertheim's theory as presented by Segura, et al. [30]. The main difference with the latter is in the way the cavity correlation function is calculated, where Bymaster and Chapman used a weighted density in the form of WDA. First, the association functional is obtained from

$$
\beta F^{\mathrm{as}}[\boldsymbol{\rho}(\mathbf{r})]=\int d \mathbf{r} \sum_{i}^{n} \rho_{i} \sum_{A_{i}}^{\left\{A_{i}\right\}}\left(\ln \chi_{A_{i}}(\mathbf{r})-\frac{\chi_{A_{i}}(\mathbf{r})}{2}+\frac{1}{2}\right) .
$$

The fraction of non-bonded particles is given by

$$
\chi_{A_{i}}(\mathbf{r})=\left(1+\int d \mathbf{r}^{\prime} \sum_{j}^{n} \rho_{j}\left(\mathbf{r}^{\prime}\right) \sum_{B_{j}}^{\left\{B_{j}\right\}} \chi_{B_{j}}\left(\mathbf{r}^{\prime}\right) \Delta^{A_{i} B_{j}}\left(\mathbf{r}, \mathbf{r}^{\prime}\right)\right)^{-1}
$$

and the association strength for iSAFT is

$$
\Delta^{A_{i} B_{j}}\left(\mathbf{r}, \mathbf{r}^{\prime}\right)=K^{\mathrm{iSAFT}} f^{A_{i} B_{j}} y_{i j}^{\sigma \sigma}\left(\mathbf{r}, \mathbf{r}^{\prime}\right) .
$$

The bonding volume is calculated with $K^{\mathrm{iSAFT}}=0.5 \pi\left(1-\cos \theta_{c}\right)^{2} \sigma^{2}\left(r_{c}-\sigma\right)$ in order to be consistent with the evaluation of the integral for the fraction of non-bonded particles [10]. The cavity correlation function of the inhomogeneous fluid is approximated as a geometric mean of the values of its bulk counterpart evaluated at contact

$$
y_{i j}^{\sigma \sigma}\left(\mathbf{r}, \mathbf{r}^{\prime}\right)=\left(y_{i j}^{\sigma \sigma}(\overline{\boldsymbol{\rho}}(\mathbf{r})) \times y_{i j}^{\sigma \sigma}\left(\overline{\boldsymbol{\rho}}\left(\mathbf{r}^{\prime}\right)\right)\right)^{1 / 2} .
$$

The expression for the cavity correlation function of the bulk fluid can be found in Ref. [8]. The weighted density shown in Eq. (18) for each component can be obtained from

$$
\bar{\rho}_{i}(\mathbf{r})=\frac{3}{4 \pi \sigma_{i}^{3}} \int d \mathbf{r}^{\prime} \rho_{i}\left(\mathbf{r}^{\prime}\right) \Theta\left(\sigma_{i}-\left|\mathbf{r}-\mathbf{r}^{\prime}\right|\right) .
$$

The associating WDA (aWDA) approximation presented in this work makes use of the same weighted density shown in Eq. (19). We calculate the association functional 
with

$$
\beta F^{\mathrm{as}}[\boldsymbol{\rho}(\mathbf{r})]=\int d \mathbf{r} f^{\mathrm{as}, \mathrm{b}}(\overline{\boldsymbol{\rho}}(\mathbf{r})) .
$$

Where $f^{\mathrm{as}, \mathrm{b}}$ is the bulk free energy density of the associating fluid as a function of a series of weighted densities $\overline{\boldsymbol{\rho}}(\mathbf{r})=\left\{\bar{\rho}_{1}(\mathbf{r}), \bar{\rho}_{2}(\mathbf{r}), \ldots, \bar{\rho}_{n}(\mathbf{r})\right\}$. Therefore, the free energy density reads

$$
f^{\mathrm{as}, \mathrm{b}}(\overline{\boldsymbol{\rho}}(\mathbf{r}))=\sum_{i}^{n} \bar{\rho}_{i}(\mathbf{r}) \sum_{A_{i}}^{\left\{A_{i}\right\}}\left(\ln \chi_{A_{i}}(\overline{\boldsymbol{\rho}}(\mathbf{r}))-\frac{\chi_{A_{i}}(\overline{\boldsymbol{\rho}}(\mathbf{r}))}{2}+\frac{1}{2}\right),
$$

where we calculate the fraction of non-bonded species as

$$
\chi_{A_{i}}(\overline{\boldsymbol{\rho}}(\mathbf{r}))=\left(1+\sum_{j}^{n} \bar{\rho}_{i}(\mathbf{r}) \sum_{B_{j}}^{\left\{B_{j}\right\}} \chi_{B_{j}}(\overline{\boldsymbol{\rho}}(\mathbf{r})) \Delta^{A_{i} B_{j}}(\overline{\boldsymbol{\rho}}(\mathbf{r}))\right)^{-1} .
$$

For our implementation the association strength is given by

$$
\Delta^{A_{i} B_{j}}(\overline{\boldsymbol{\rho}}(\mathbf{r}))=K f^{A_{i} B_{j}} y_{i j}^{\sigma \sigma}(\overline{\boldsymbol{\rho}}(\mathbf{r})),
$$

where the cavity correlation function $y_{i j}^{\sigma \sigma}$ is simply evaluated at the weighted densities in $\mathbf{r}$ similar to Eq. (18) and (19). At last, the constant for the volume of association $K^{\text {aWDA }}$ is calculated in the same way as aFMT with Eq (14).

In all the approaches presented, the fraction of non-bonded particles $\chi_{A i}$ are obtained in an iterative damped successive substitution fashion. However, in comparison the functionals aFMT and aWDA require only the local value of its weighted densities, whereas for iSAFT the values of $\chi_{A i}$ depend on the neighbouring values through the integral over coordinate $\mathbf{r}^{\prime}$.

To obtain the equilibrium density profile the functional derivatives of $F[\boldsymbol{\rho}(\mathbf{r})]$ must be calculated. The functional derivative of the ideal contribution is

$$
\beta \frac{\delta F^{\mathrm{id}}[\boldsymbol{\rho}(\mathbf{r})]}{\delta \rho_{j}(\mathbf{r})}=\ln \rho_{j}(\mathbf{r}) .
$$

The combination of Eq. (6) with (24) leads to the following Euler-Lagrange equation that can be solved with a Piccard iteration scheme

$$
\rho_{j}(\mathbf{r})=\exp \left(\beta \mu_{j}-\beta \frac{\delta F^{\operatorname{ex}}[\boldsymbol{\rho}]}{\delta \rho_{j}(\mathbf{r})}-\beta V^{\operatorname{ext}}(\mathbf{r})\right) .
$$

For the minimization of the grand potential functional in slit-like pores, Eq. (25) can be discretized in $z$, the coordinate orthogonal to the walls. In our calculations we found that a grid spacing of $0.01 \sigma$ and the trapezoidal rule for the integrals were adequate to attain good precision in the final density profile. 


\section{Results and discussion}

We present results of confined associating hard spheres with different number of bonding sites following the schemes depicted in Fig. 1. The three classical DFT functionals described in the Section 2 are compared to Monte Carlo simulations of the corresponding systems confined in slit-like pores taken from literature for a wide range of conditions. [30-32, 39, 42]. Specifically, we tested systems of pure associating fluids and mixtures of hard spheres with associating fluids close to a single hard wall [30$32,39]$, and associating fluids confined in narrow pores where only one or two layers of particles can be formed [42].

To make a fair comparison between the models and molecular simulation results, we take care of using the same values of the parameters used in the simulations. For all results, the diameter of hard spheres is taken as $\sigma=1$. The results are presented for different values of the dimensionless temperature $T^{*}=k_{B} T / \varepsilon^{A B}$, the dimensionless density $\rho^{*}=\rho \sigma^{3}$ and the resulting equilibrium density profiles are plotted as the reduced density $\rho^{*}(z) / \rho_{\mathrm{b}}^{*}$ versus the dimensionless distance from the wall $z^{*}=z / \sigma$.

Given the differences between the associating functionals presented here, the definition of the associating volume must be selected accordingly to ensure that the calculation of the fraction of non-bonded species and the Helmholtz free energy for all three functionals is the same in the bulk limit $\rho(\mathbf{r}) \rightarrow \rho^{\text {bulk }}$, e.g. see Eqs. (12), (16) and (22). The associating limits $r_{c}$ and $\theta_{c}$ explained in Section 2 are used to calculate the volume available for association according to $[6,10]$

$$
\kappa^{A B}=\left\{\begin{array}{ll}
K^{\mathrm{aFMT}}=K^{\mathrm{aWDA}}, & \pi\left(1-\cos \theta_{c}\right)^{2} \sigma^{2}\left(r_{c}-\sigma\right) \\
K^{\mathrm{iSAFT}}, & 0.5 \pi\left(1-\cos \theta_{c}\right)^{2} \sigma^{2}\left(r_{c}-\sigma\right)
\end{array} .\right.
$$

The expressions shown here are consistent with the molecular simulations results obtained by Segura et al. [30-32, 39], and have also been used together with classical DFT in the study of associating fluids $[6,10]$. Additionally, the factor 0.5 appearing in the expression for $K^{\mathrm{iSAFT}}$ is added to account for the range of integration of the iSAFT functional [10].

Once the equilibrium density profile has been determined, the fraction of monomers as a function of the distance from the wall can be calculated from

$$
\chi_{A_{i}}(z)=\left(1+\Delta^{A_{i} B_{j}}\left(\rho_{\mathrm{b}}\right) \int d z^{\prime} \sum_{j}^{n} \rho_{j}\left(z^{\prime}\right) \sum_{B_{j}}^{\left\{B_{j}\right\}} \chi_{B_{j}}\left(z^{\prime}\right)\right)^{-1} .
$$

Where $\Delta^{A_{i} B_{j}}\left(\rho_{\mathrm{b}}\right)$ is the association strength evaluated at bulk conditions and with associating volume $K=0.5 \pi\left(1-\cos \theta_{c}\right)^{2} \sigma^{2}\left(r_{c}-\sigma\right)$ for the three functionals. We use this procedure for the calculation of the fraction of non-bonded species as shown in Eq. (27) following the work of Segura et al. [30] and $\mathrm{Yu}$ and $\mathrm{Wu}$ [6]. Moreover, as all association sites of each particle are considered equal, the fraction of monomers in a given position is then given by

$$
\chi_{0}(z)=\left(\chi_{A_{i}}(z)\right)^{M}
$$

with $M$ as the number of association sites. 


\subsection{Scheme $1 A$}

In this section we study the performance of classical DFT in the determination of the density distribution for fluids with one association site. The results are shown in Figs. 2 and 3 for two average bulk densities of approximately $\rho_{\mathrm{b}}^{*}=0.2$ and $\rho_{\mathrm{b}} *=0.49$ at two temperatures each. Furthermore, in Fig. 4 we present results for the fraction of non-bonded particles for some of these cases.

As we are only taking into account volume exclusion effects and association in our implementation we expect to observe competition between these two contributions. Depending on the conditions of each of the systems studied, one contribution may predominate over the other and these can be readily observed as we vary the overall density and the dimensionless temperature. Fig. 2a shows a typical behavior of a density profile of a system mainly dominated by the hard sphere contribution, with high density values close to the wall and a decrease to the bulk value as the distance from the wall increases $[3,5]$. On the other hand, in Fig. $2 \mathrm{~b}$ the system has approximately the same overall density but at a considerably lower dimensionless temperature, which leads to an increase in the degree of association. The behavior of this system is largely influenced by associating effects and only a small amount of monomers remain free, e.g. see Fig. 4 (diamonds). The latter implies that the formation of dimers or clusters is favored, causing a depletion in the density profile close to the wall. This effect has been described before in previous studies of inhomogeneous associating fluids $[6,10,29-32,39]$, and consequentially in the study of inhomogeneous polymeric fluids that include a chain formation contribution to the free energy functional $[7,8]$. Because of the larger size of the clusters, there is a restriction in the configurations available for the clusters close to the wall in comparison to the bulk, which causes depletion or desorption. Generally, the agreement between all the functionals and simulations is very good. All three functionals converge to the same value of the density at contact to the wall, which agrees with the wall theorem [1], and far from the wall they reach the bulk densities. In Fig. 2a the peak corresponding to the first coordination shell at around $z^{*}=1.5$ is well captured by iSAFT, whereas aMFT and aWDA give a smoother transition but still in agreement with the simulation results. Fig. $2 \mathrm{~b}$ shows discrepancies for aFMT and aWDA when compared with simulations. aFMT overpredicts depletion and both aFMT and aWDA underpredict the transition from depletion to adsorption at $z^{*}=1.5$. For this case only iSAFT is able to accurately capture the behavior of the density profile.

In Fig. 3 there are two cases with larger values of the average bulk density and the packing effects are more pronounced. In both cases we observe a high accumulation of particles close to the wall and a slightly higher degree of layering that are perfectly captured by all the functionals. With a decrease in the dimensionless temperature in Fig. 3b, we observe a decrease in the density at contact, but the formation of clusters is not large enough to cause depletion at the wall as the hard sphere contribution is dominant, e.g. see Fig. 4 (squares). For these two cases iSAFT shows an excellent agreement with simulation results. aFMT and aWDA show also a very good agreement with slight deviations. It is known that some of the implementations of the associating functional with WDA lead to a high degree of layering at high densities $[6,10]$. However, with our implementation we observe that the layering of the density distribution falls very well between the margin of errors of the simulation results [31]. Additionally in Fig. 3b, aFMT underpredicts the layering shown by the density profile whereas aWDA tends to extend it further from the wall.

The profiles of the fraction of monomers are plotted in Fig. 4 . For all the cases it is 


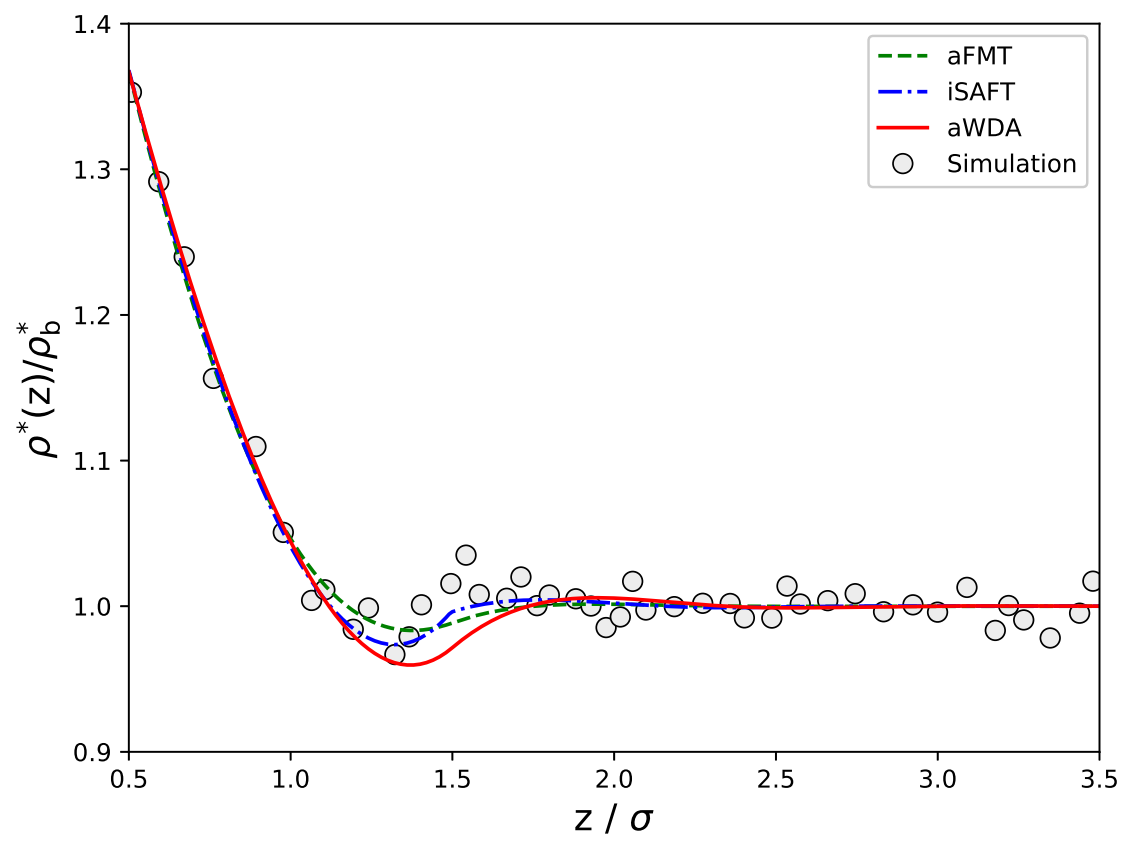

(a)

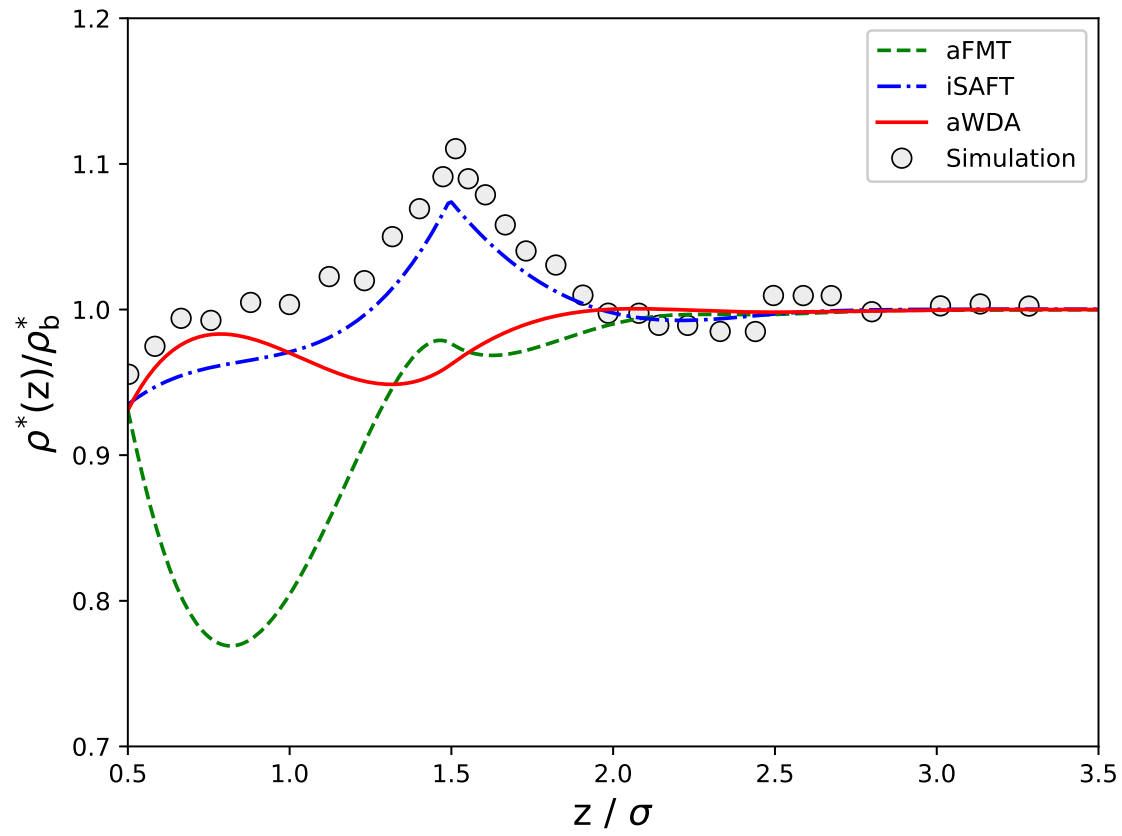

(b)

Figure 2. Equilibrium density profiles of a dimerizing hard sphere $(1 \mathrm{~A})$ close to a wall calculated with classical DFT with three different association functionals at (a) $\rho_{\mathrm{b}}^{*}=0.1984$ and $1 / T^{*}=7.0$, (b) $\rho_{\mathrm{b}}^{*}=0.1999$ and $1 / T^{*}=14.0$. Symbols are results from Monte Carlo simulations from Ref. 31 . 
observed that the bonding of particles decreases close to the wall, as the wall blocks some of the associating sites available for bonding and configurations available for cluster formation. As expected, the degree of association increases together with bulk density and with decreasing temperature. The results for $\chi_{0}(z)$ for all three functionals are obtained with Eq. (27). It is seen that aFMT, iSAFT and aWDA agree very well with the simulation results and the differences from the density profiles do not affect the distribution of the fraction of monomers. It is important to note that $\chi_{0}(z)$ shown in this figure is calculated after the minimization of the grand potential $[6,31]$ and results are similar because for the three functionals the association strength is approximated in the same way. The discontinuities in slope at $z^{*}=1.5$ correspond to the first coordination shell [1], and are caused because during calculation of Eq. (27), the integrand vanishes at $z^{*}<0.5$.

\subsection{Scheme 2B}

In this section we study the behavior of associating fluids with two bonding sites close to hard walls. Associating fluids with more than one bonding sites are able to polymerize, or to form larger clusters in comparison with fluids with one bonding sites (1A) as discussed in Section 3.1, which can be translated into larger depletion effects in the density distribution close to the wall. An example can be clearly observed in Fig. 5, which is at similar conditions as Fig. 2 but for the two sites case. Here, the degree of depletion is larger than for $1 \mathrm{~A}$ and it extends further from the wall until about $z^{*}=3.5$. The results obtained with the three functionals agree very well with simulation results only with small differences that are within the range of errors as reported in Ref. 31. Even though the three functionals predict the same value for the density at contact, aFMT describes higher depletion close to the wall in comparison with the simulations, whereas iSAFT and aWDA show better agreement in this regard. However, it is difficult to formulate a strong conclusion based on this result as Segura et al. [31] discussed that there was evidence that the simulation did not reached equilibrium therefore causing a large standard deviation.

Fig. 6 depicts the transition from a system mainly dominated by association to another where the packing effects close to the wall are greater as the bulk density and temperature increase. Particularly, results obtained with classical DFT do not agree very well with the simulation in comparison with the other cases. There is a slight discrepancy in the density at contact with the simulation but all functionals converge to the same value. As the errors were not reported for this case, we cannot fully assessed the differences between the functionals but in a qualitatively way simulations and classical DFT agree with each other. In terms of the difference between the functionals results are quite simuilar to Fig. 3, aFMT shows larger depletion close to the wall than the other two, and aWDA shows a larger degree of oscillations that extends to about $z^{*}=2.5$.

A system with higher density and temperature is shown in Fig. 7. Here associating effects are mitigated and the system is mainly dominated by packing effects. The agreement between molecular simulation and classical DFT is excellent and all three functionals perform similarly, being WDA slightly superior to the other functionals in describing the layering of the density profile.

The fraction of monomers for hard sphere fluids with two associationg sites are plotted in Fig. 8. In general there is good agreement between simulation results and classical DFT. However, it is observed that there are larger deviations between simu- 


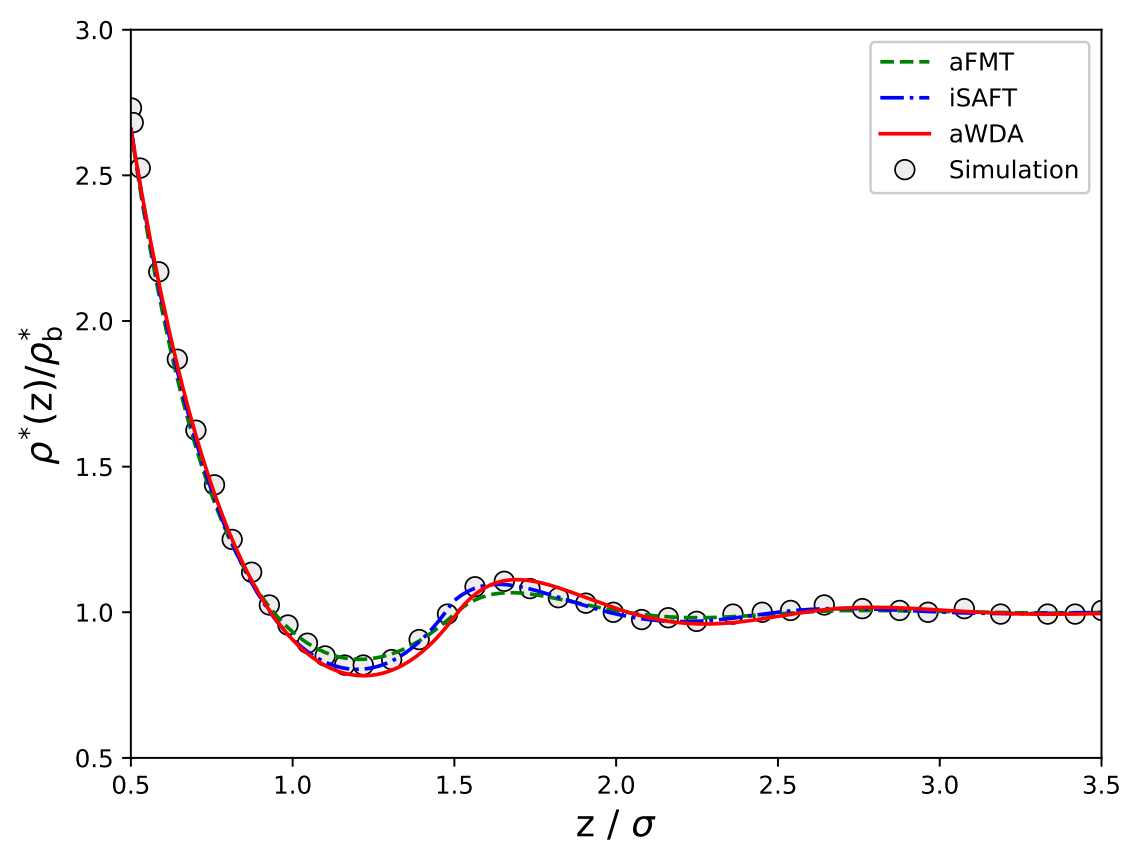

(a)

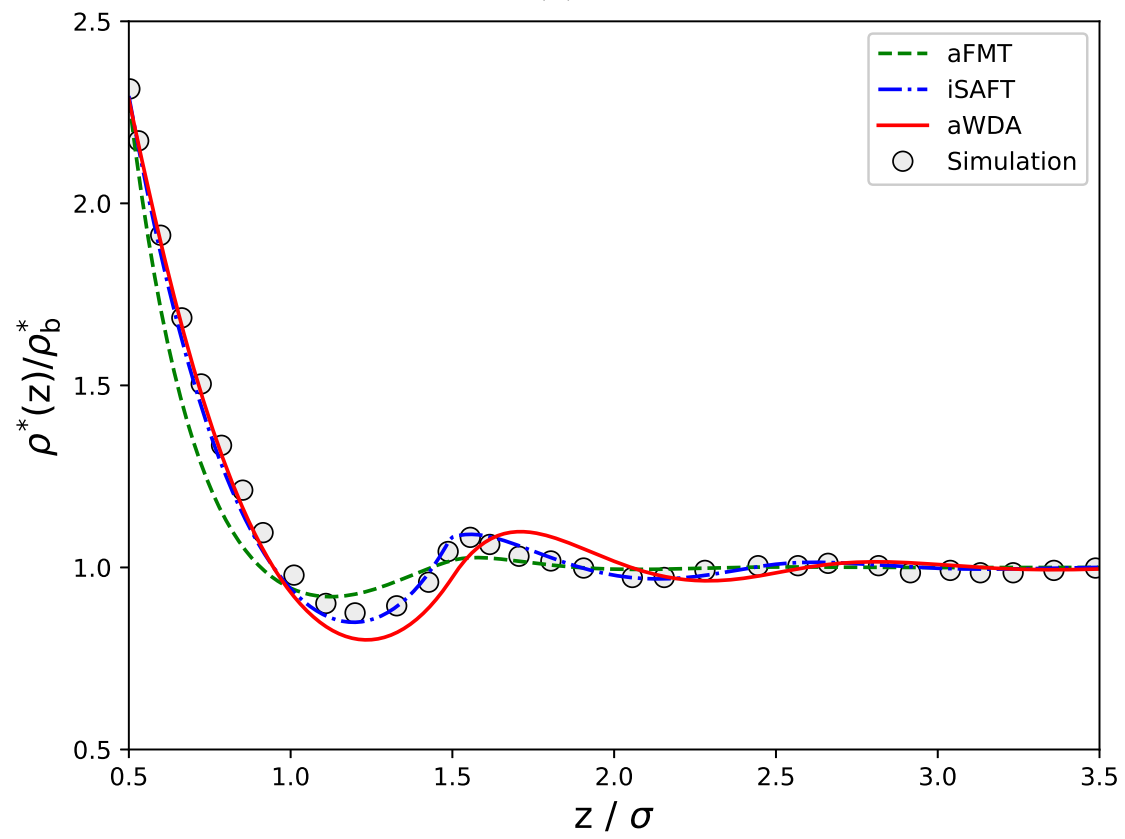

(b)

Figure 3. Same as Fig. 2 for (a) $\rho_{\mathrm{b}}^{*}=0.4855$ and $1 / T^{*}=7.0$, (b) $\rho_{\mathrm{b}}^{*}=0.4868$ and $1 / T^{*}=11.0$. 


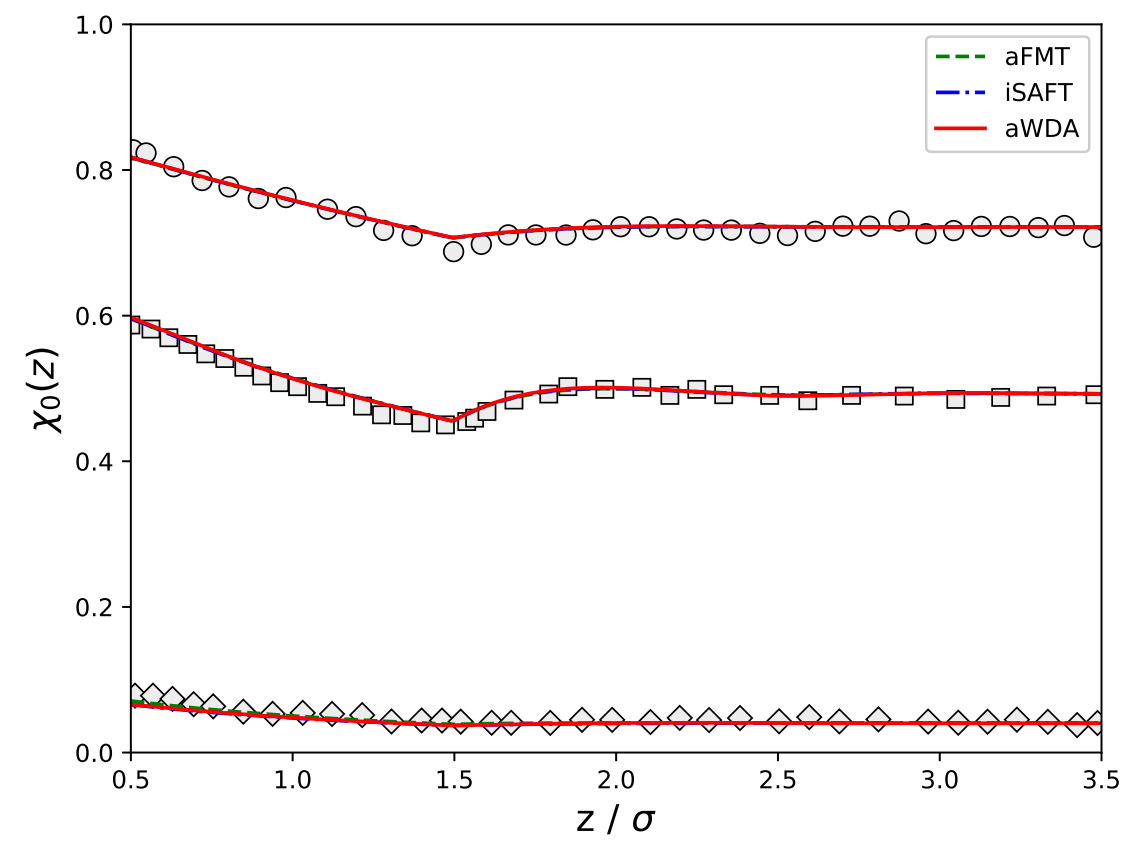

Figure 4. Fraction of monomers for the associating hard spheres (1A) close to a hard wall. From top to bottom $\rho_{\mathrm{b}}^{*}=0.1984$ and $1 / T^{*}=7.0$ (circles), $\rho_{\mathrm{b}}^{*}=0.4855$ and $1 / T^{*}=7.0$ (squares), and $\rho_{\mathrm{b}}^{*}=0.1999$ and $1 / T^{*}=14.0$ (diamonds). Symbols are results from Monte Carlo simulations from Ref. 31

lation and theory closer to the wall and also as the fraction of monomers increases.

\subsection{Scheme $4 C$}

We describe the structure of a fluid with four bonding sites following the scheme $4 \mathrm{C}$ as previously described. The location of the sites in the sphere is intended to simulate the electron acceptors and donnors sites of the water molecule able to form hydrogen bonds. Fig. 9 shows the first two sets of density profiles evaluated at two temperatures with an average bulk density of $\rho_{\mathrm{b}}^{*}=0.2$ for the water-like model. In panel (a) at high temperature or low bonding energy there is adsorption close to wall, a slight decrease in density around $z^{*}=1.25$ and then the density profile stabilizes to its bulk value. The results obtained with classical DFT agree very well with the simulation, specially WDA and iSAFT which describe the first density valley with very good precision. On the other hand, aFMT overpredicts the density value before reaching the bulk density. The simulation results show oscillations at rather large distances from the wall, but are kept steady around the bulk value reported [30]. For panel (b), the decrease in temperature leads to an increase in association which is observed by the presence of desorption close to the wall. The three functionals predict the same value for the density at contact, which slightly differs from the value shown by the simulation results. aFMT and iSAFT give very similar results, whereas results from WDA agree better with the simulation.

As we increase the bulk density, we observe a more pronounce competition between association and volume exclusion. Association of particles increases together with density and association energy, or lower temperatures. Fig. 10 shows two examples for this case, in panel (a) we clearly observe how the packing effect dominate over association, as we have high densities close to the wall and layering up to two diameters. There is 


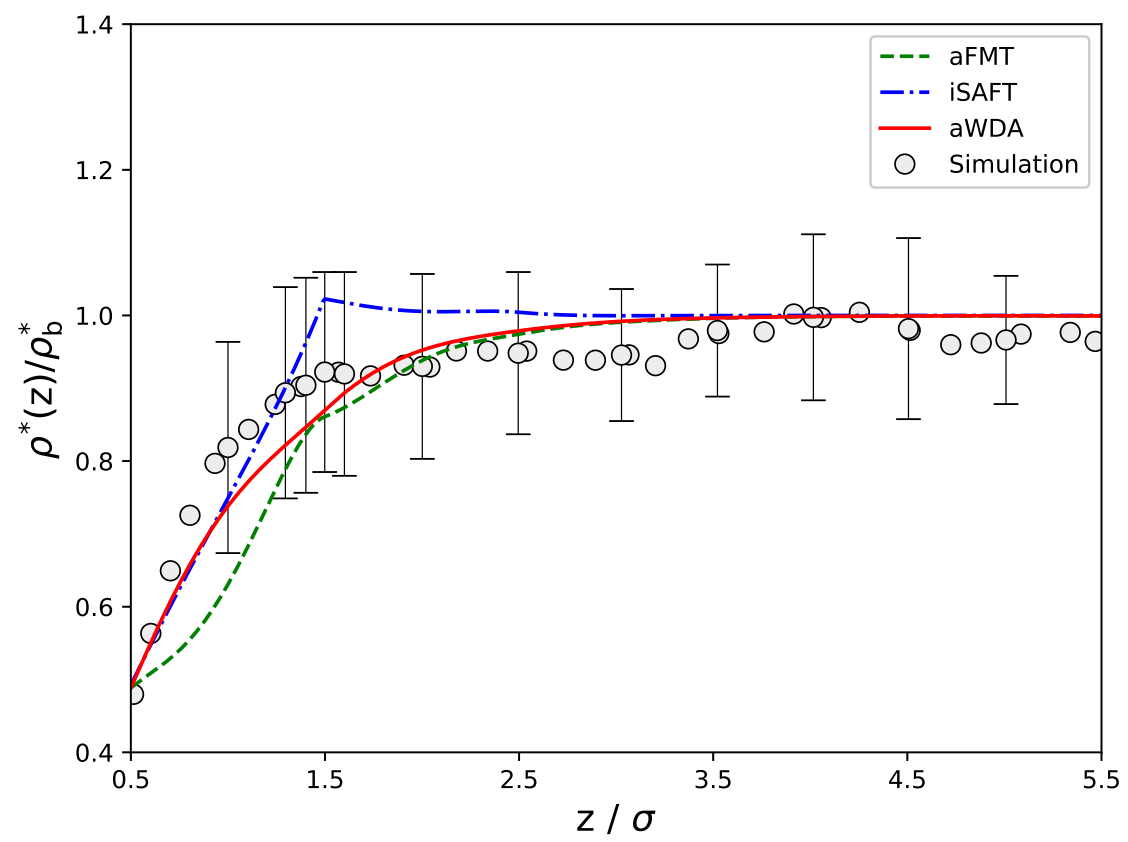

Figure 5. Density distribution of a hard sphere with two association sites (2B) close to a wall calculated with classical DFT with three different association functionals at $\rho_{\mathrm{b}}^{*}=0.2084$ and $1 / T^{*}=11.0$. Symbols and errors are results from Monte Carlo simulations digitalized from Ref. 31.

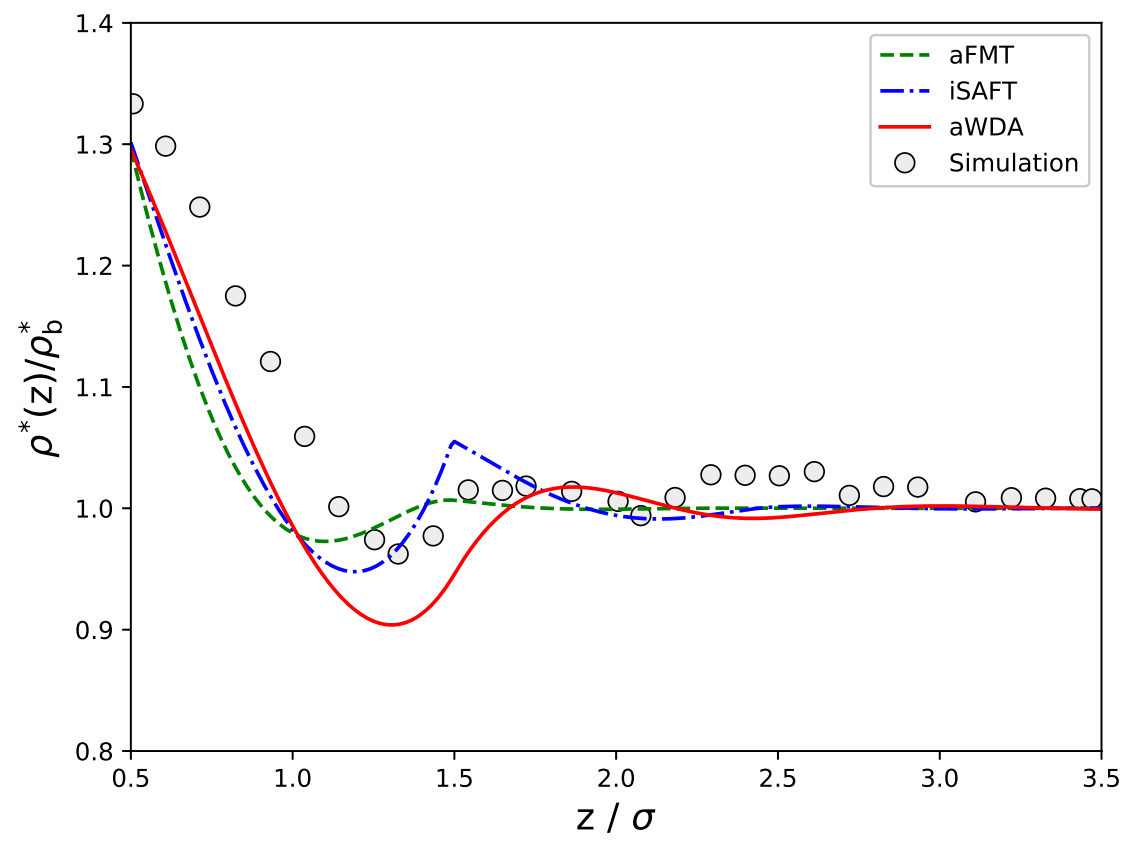

Figure 6. Same as Fig. 5 for $\rho_{\mathrm{b}}^{*}=0.3449$ and $1 / T^{*}=8.0$. 


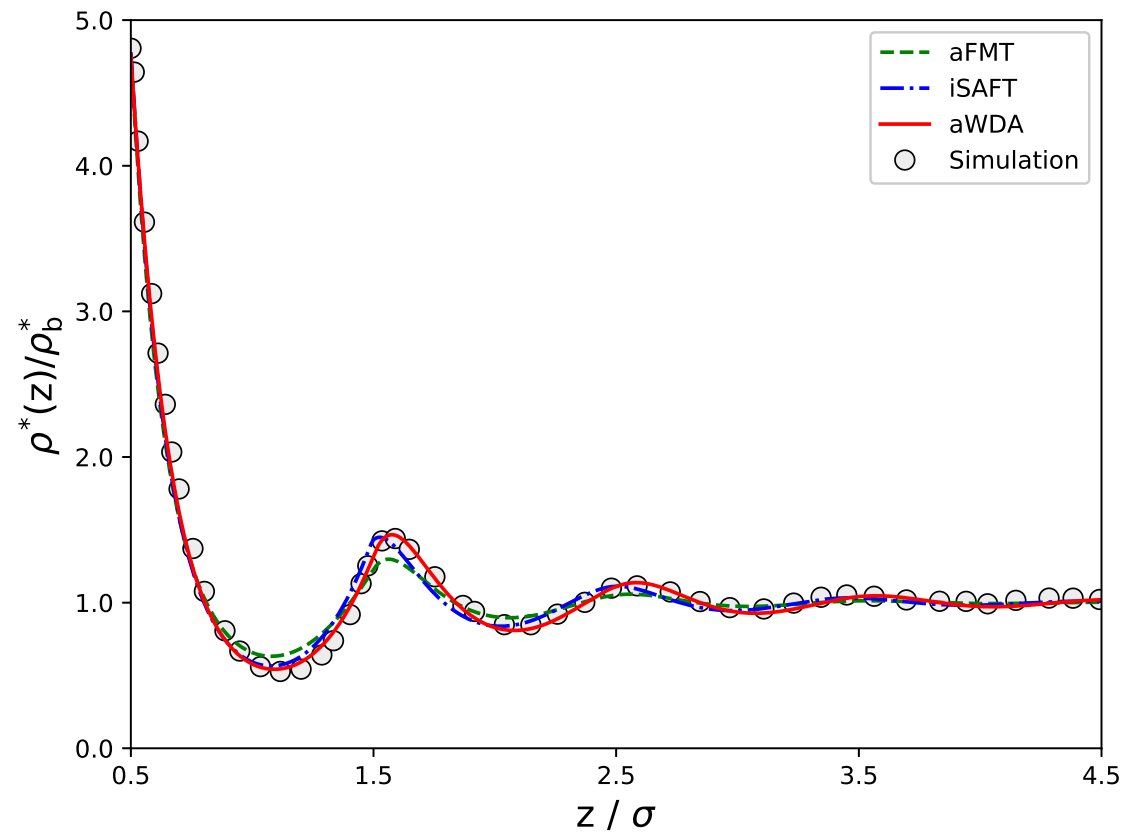

Figure 7. Same as Fig. 5 for $\rho_{\mathrm{b}}^{*}=0.7177$ and $1 / T^{*}=6.0$.

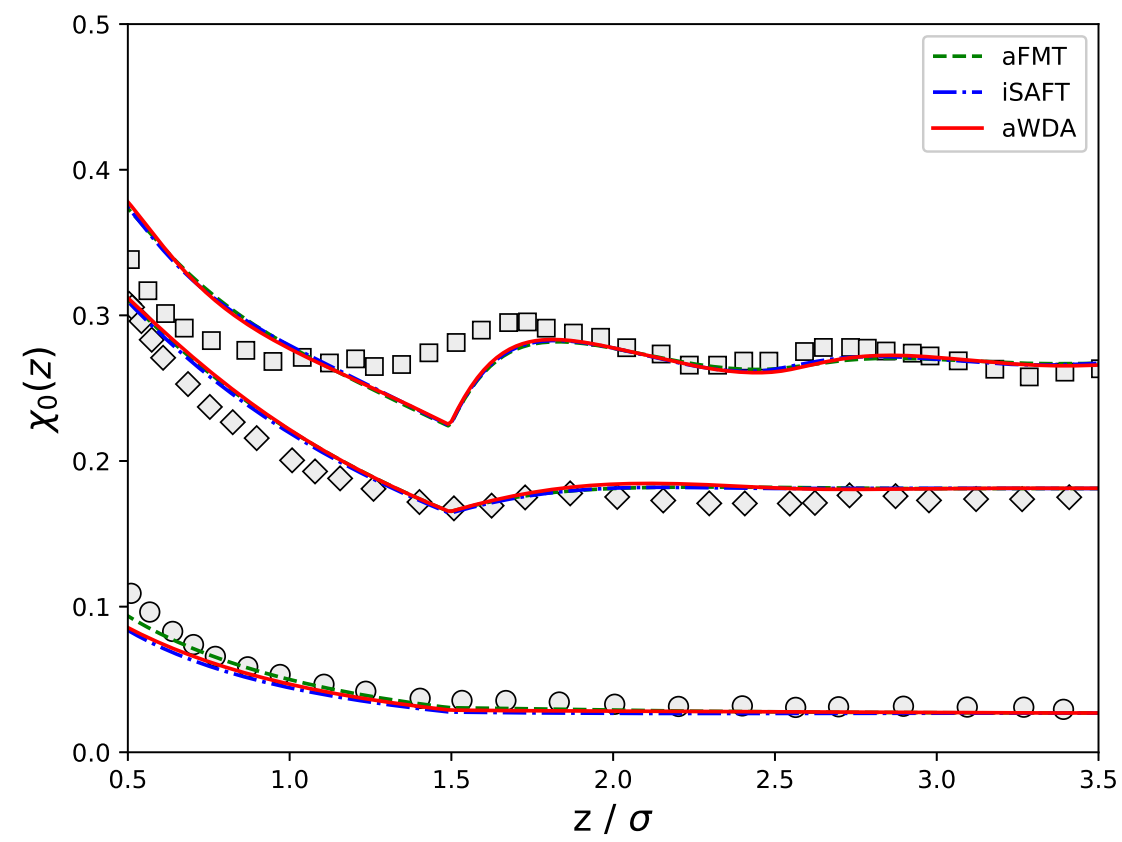

Figure 8. Comparison of the fraction of monomers for the fluid with two association sites close to a hard wall. From top to bottom at $\rho_{\mathrm{b}}^{*}=0.7177$ and $1 / T^{*}=6.0$ (squares), at $\rho_{\mathrm{b}}^{*}=0.3449$ and $1 / T^{*}=8.0$ (diamonds), and at $\rho_{\mathrm{b}}^{*}=0.2084$ and $1 / T^{*}=11.0$ (circles). Symbols are results from Monte Carlo simulations from Ref. 31 . 


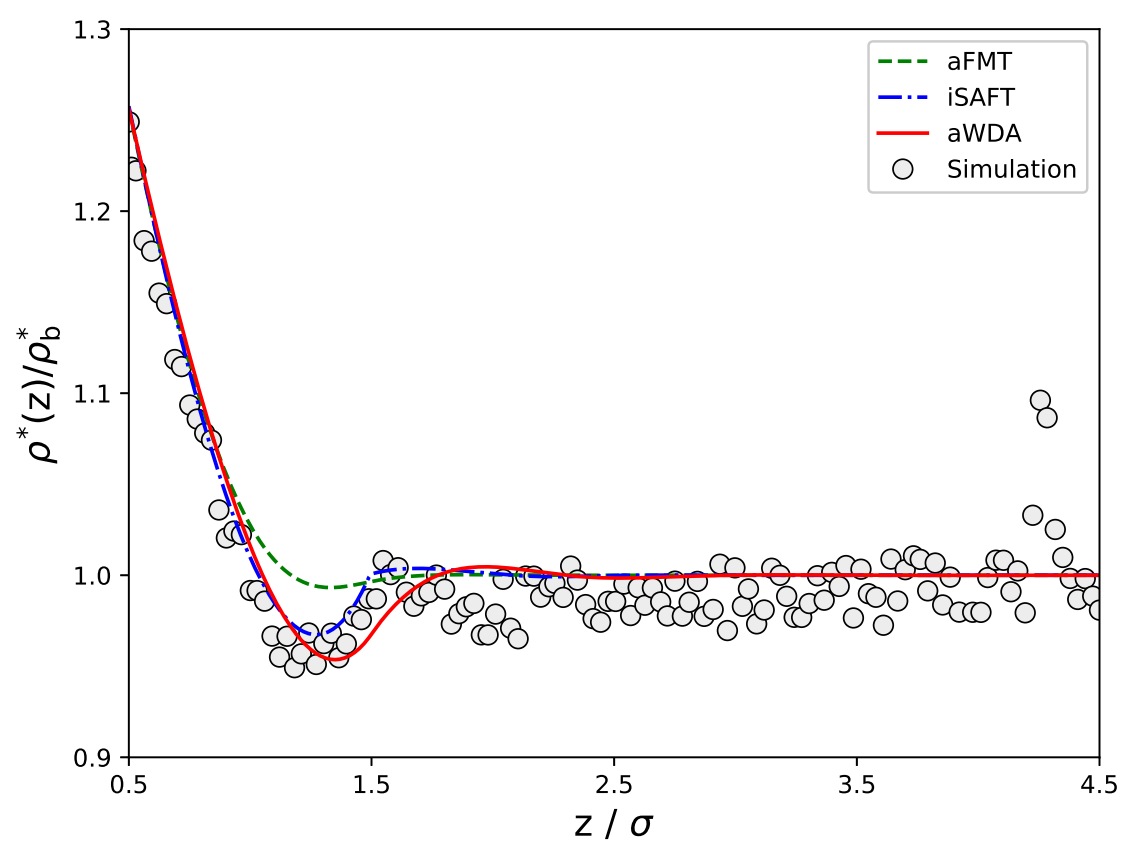

(a)

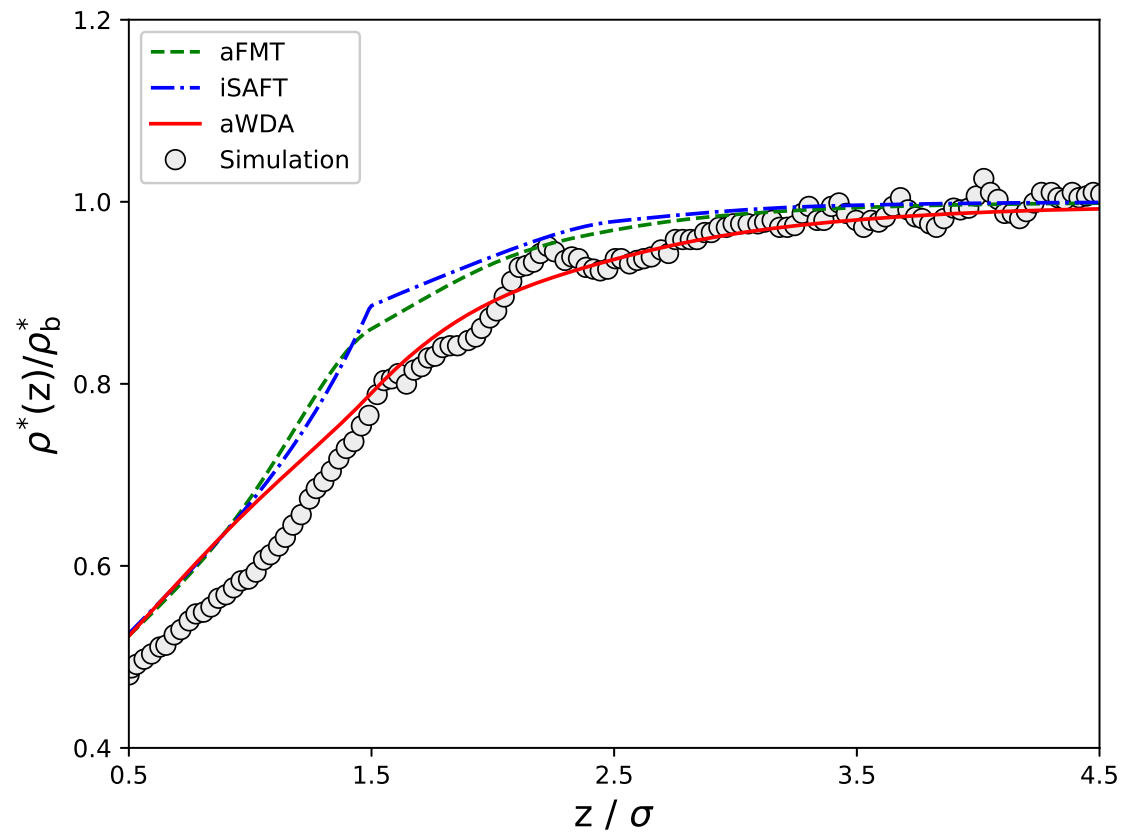

(b)

Figure 9. Density profile of a hard sphere fluid with four association sites $(4 \mathrm{C})$ as a function of the distance from a hard wall at (a) $\rho_{\mathrm{b}}^{*}=0.1994$ and $1 / T^{*}=5.0$, and (b) $\rho_{\mathrm{b}}^{*}=0.2112$ and $1 / T^{*}=7.0$. Symbols are results from Monte Carlo simulations from Ref. 30. 
good agreement of this behavior between simulations and theory, aWDA and iSAFT tend to slightly exaggerate oscillation in density just before the first coordination shell, whereas aFMT shows a smoother transition in the density profile. Panel (b) shows the interesting case that gives evidence to the shift in the density profile from a system mainly dominated by volume exclusion and collisions of particles towards a state where cluster formation prevents adsorption of the associated particles. The three functionals predict this event in good agreement with simulation, but it is only perfectly capture by aWDA in the whole section of the slit. These simulations results were taken from the work of Patrykiejew et al. [39] as was pointed out that the results for these conditions presented in Ref. 30 where not correct due to difficulties in convergence of the Monte Carlo procedure.

Panels (a) and (b) from Fig. 11 show two equilibrium density profiles at relatively high densities for the same association energy. For these two systems, the density profiles show layering far from the wall up to $z^{*}=4.5$ characteristic of high packing effects. The three functionals give fairly similar results and are in good agreement with simulations. For Fig. 11a aWDA and iSAFT slightly overpredict the peak corresponding to the first coordination shell, whereas aFMT underpredicts it and smoothes out closer to the wall. Both, the position of the first peak and the density value at contact are in agreement among the functionals. However, after $z^{*}>1.5$ the peaks gradually shift out of synchrony between simulations and theories. In Fig. 11b, for a density close to the hard sphere freezing point [6], there is better agreement between the three functionals as the effects of association decrease and the hard sphere term predominates. Moreover, the location of the peaks agree very well with Monte Carlo results. Although all classical DFT calculations have excellent performance, aWDA is slightly better in predicting the location and values of the layers in the density distribution. Fig. 12 shows two examples of the fraction of monomers as a function of the distance from the wall. Similar behavior as from the previous cases is found, and all functionals agree very well with the simulation results.

\subsection{Narrow pores}

So far, only results for associating fluids confined in large pores have been studied, in which the density distribution in the middle part of the pore reaches the bulk density. In this section we study the structure of this associating fluid under extreme confinement, where the size of the pure is sufficiently small to only allow from one to three layers of particles to form inside of the slit-like pore.

We study the behavior of a hard sphere fluid with four association sites confined in smooth hard walls with separations of $H=1.18 \sigma, H=2.31 \sigma$ and $H=3.02 \sigma$. The classical DFT results with the three functionals are plotted and compared with Monte Carlo simulations of the same systems [42]. In these simulations, the association between particles is only taken into account with the distance between the association sites, i.e. ignoring the angles defined in Eq. 3. Therefore, the association volume used for the following calculations was set to $K=4 \pi / 3\left(r_{c}-\sigma\right)^{3}$ with $r_{c}=1.1 \sigma[42,43]$. For the three cases, the results are shown for a total average density inside the pore of $\rho_{\mathrm{AVG}}=0.588$, which is defined as

$$
\rho_{\mathrm{AVG}}=\frac{1}{H} \int \rho(z) d z .
$$

Eq. (29) implies that the bulk density used for the classical DFT calculation must 


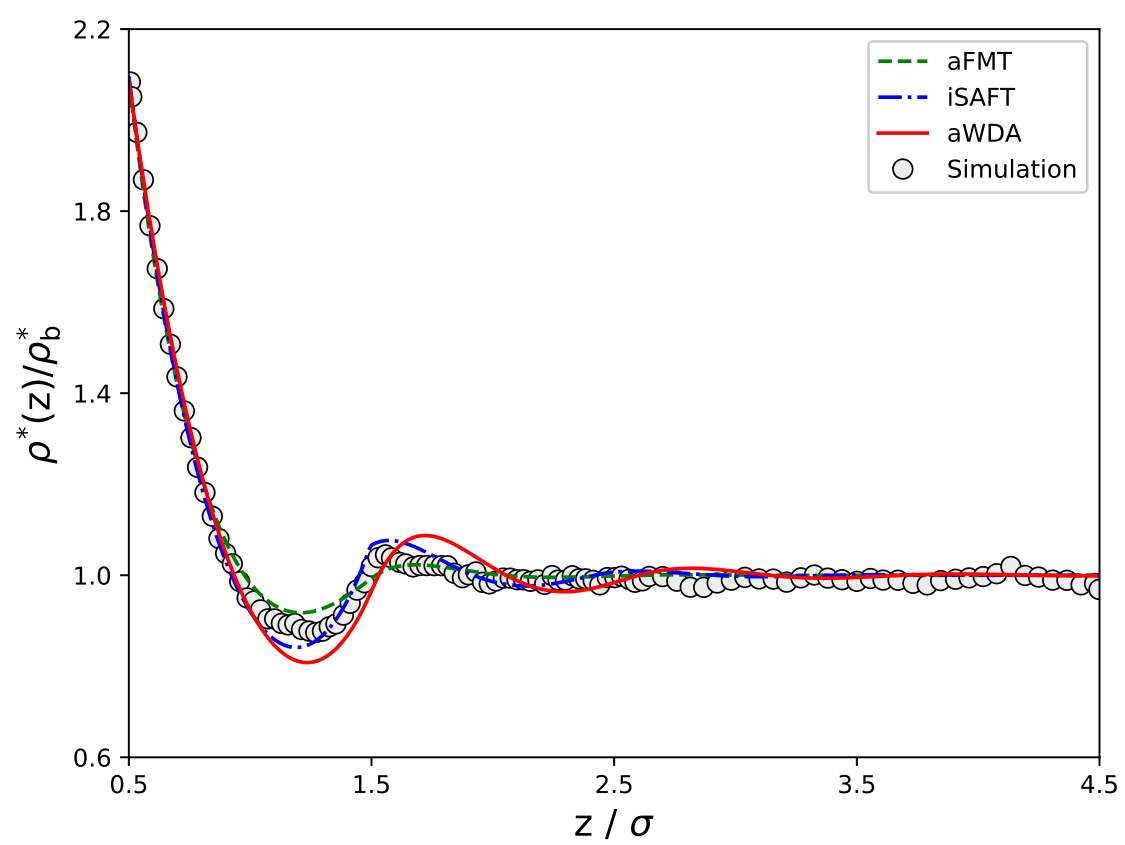

(a)

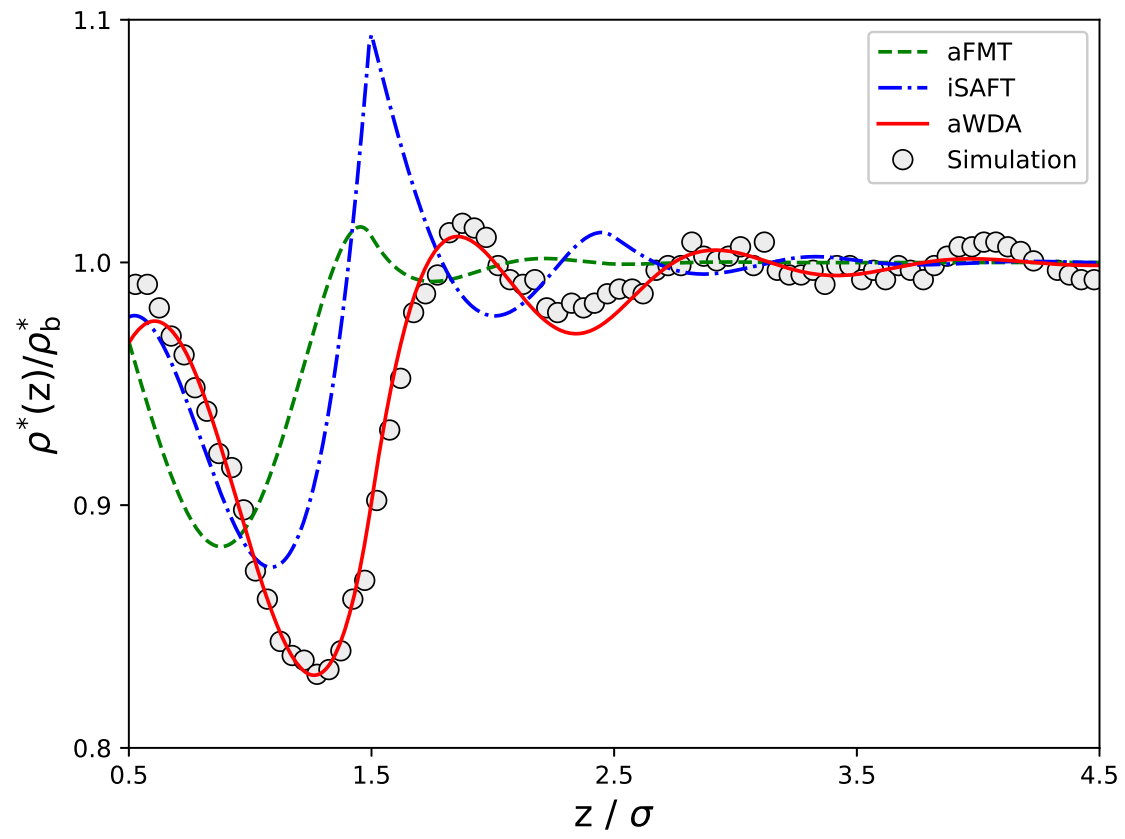

(b)

Figure 10. Comparison of the equilibrium density profile of a associating fluid with scheme $4 \mathrm{C}$ with simulation results. (a) $\rho_{\mathrm{b}}^{*}=0.4914$ and $1 / T^{*}=5.0$ simulation results from Ref. [30], and (b) $\rho_{\mathrm{b}}^{*}=0.5260$ and $1 / T^{*}=7.0$ simulations results from Ref. 39. 


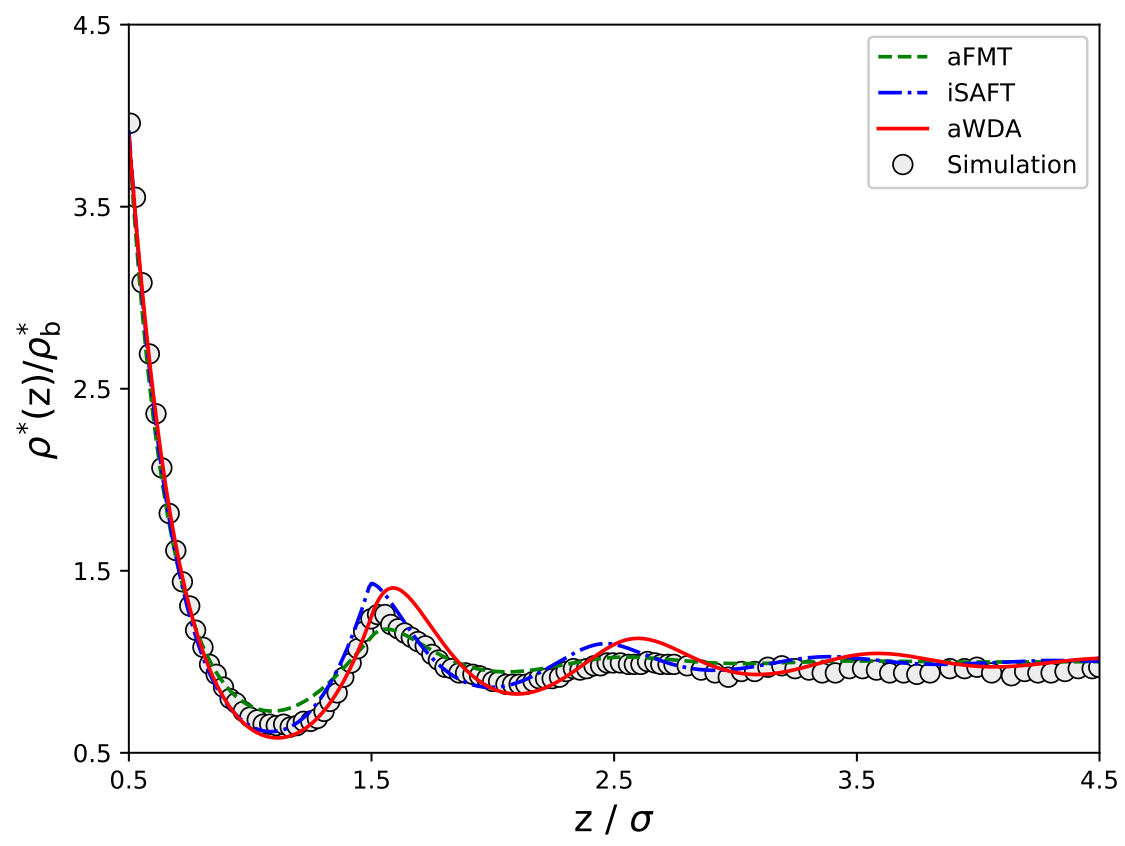

(a)

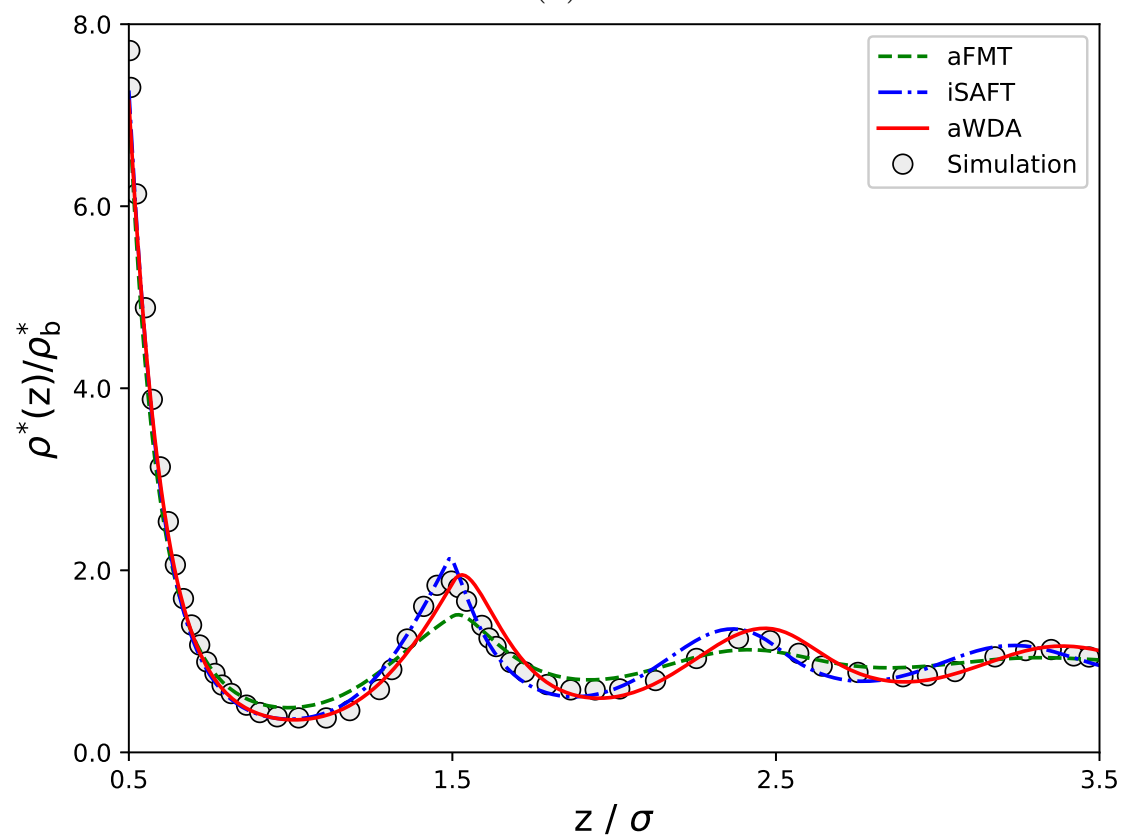

(b)

Figure 11. Equilibrium density profile of a associating fluid with four sites at (a) $\rho_{\mathrm{b}}^{*}=0.7257$ and $1 / T^{*}=5.0$, and (b) $\rho_{\mathrm{b}}^{*}=0.9036$ and $1 / T^{*}=5$.0. Simulations results from Ref. 30 are shown as symbols in both panels. 


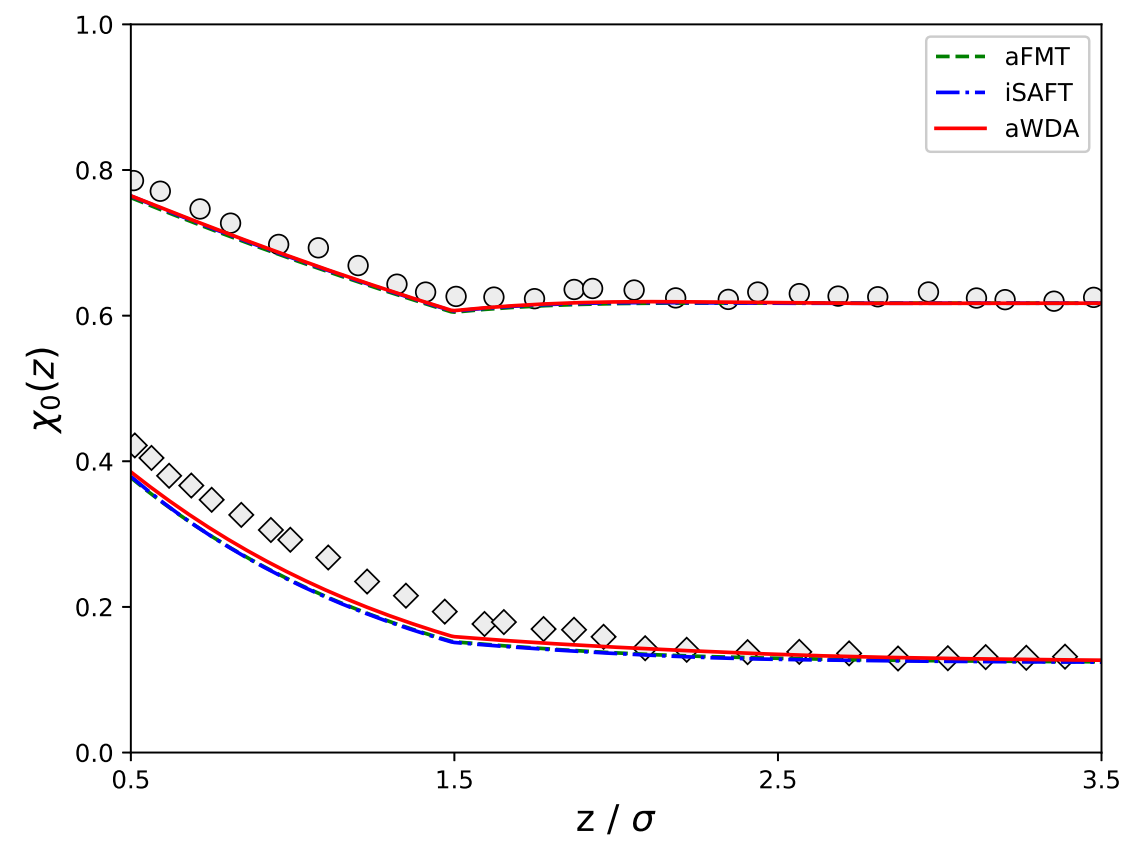

Figure 12. Comparison of the monomer fraction of the fluid with four association sites calculated with three different functionals, symbols show Monte Carlo simulation results [30]. In the top at $\rho_{\mathrm{b}}^{*}=0.1994$ and $1 / T^{*}=5.0$, and bottom $\rho_{\mathrm{b}}^{*}=0.2112$ and $1 / T^{*}=7.0$.

not necessarily be the same. In the ideal situation, in which all three functional agree perfectly with the molecular simulations, the bulk density used for the three functionals would be equivalent. In order to find the adequate bulk density that gives the desired average density in the pore after minimization of the grand potential functional, we used Brent's method, a root-finding algorithm based in root bracketing [44].

The results for the three systems in narrow pores with widths of $H=1.18 \sigma$, $H=2.31 \sigma$ and $H=3.02 \sigma$ are shown in Figs. 13, 14 and 15, respectively. For the smallest pore, there is only a small volume available for the associating particles delimited by $0.5<z^{*}<0.68$ due to volume exclusion between the particles and the walls. In comparison with the molecular simulation results, the three functionals underpredict the density at contact with the walls. aFMT shows better agreement with the simulations with higher densities close to the walls, whereas iSAFT predicts a nearly flat density distribution and aWDA gives the opposite trend with high densities in the middle of the pore and depletion close to the walls. In this conditions of extreme confinement molecular simulations show that the system is mainly dominated by exclusion effects of the hard spheres. As mentioned previously, this type of conditions in which the competition between different contributions to the Helmholtz free energy are the most difficult situations for the functionals. It is evident that this effect is well captured by aFMT. iSAFT, and especially aWDA tend to increase the effect of associating interactions which leads to a decrease in the density close to the wall.

Fig. 14 shows the system of associating particles confined in a slit with a separation between the walls of $H=2.31 \sigma$. The system shows a transition from two layers of particles inside the pore, to a system with three layers of particles as shown in Fig. 15 for $H=3.02 \sigma$. All three functionals are able to predict the transition from two to three layers in good qualitatively agreement with simulations, but predict a lower density at contact for the pore sizes and a different behavior in the density 


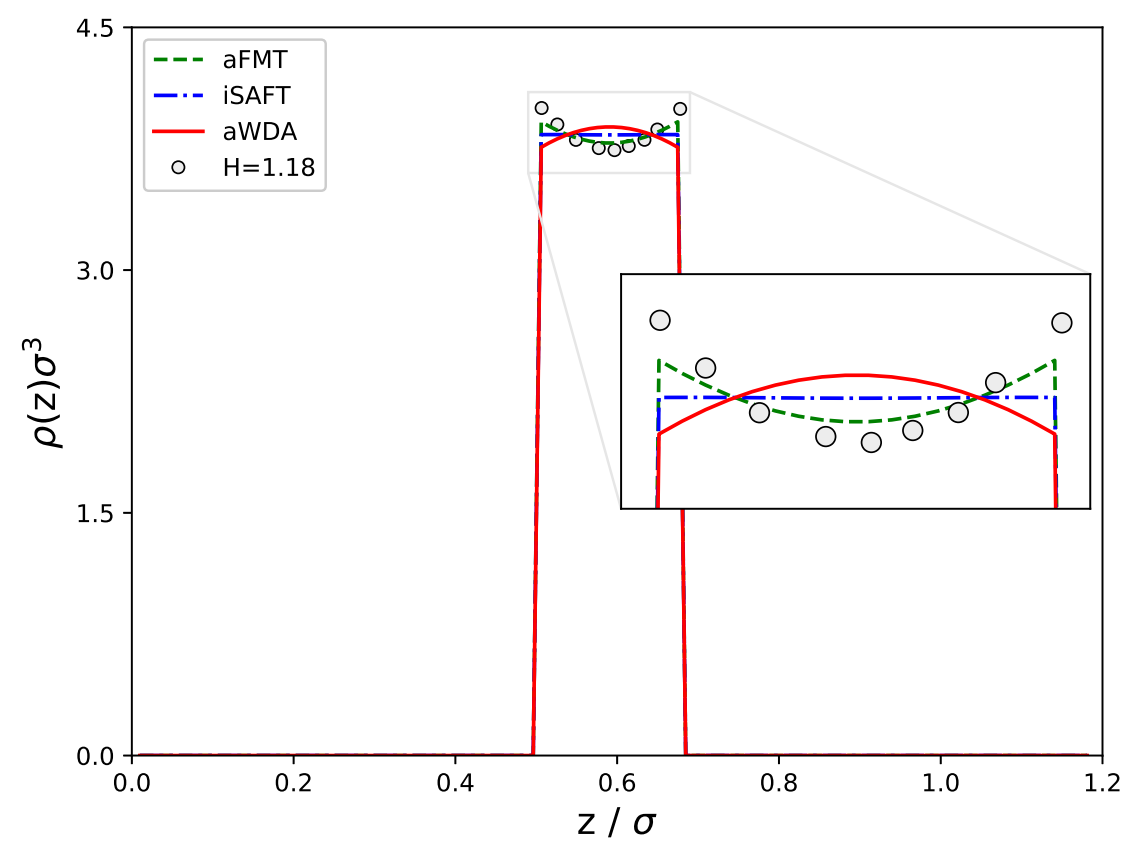

Figure 13. Density distribution of a hard sphere fluid with four association sites $\left(1 / T^{*}=8.0\right)$ confined in a slit-pore of $H=1.18 \sigma$ with an average density of $\rho_{\mathrm{AVG}}=0.588$ inside the pore. Lines are results obtained with classical DFT and three different association functionals and symbols are results from molecular simulations [42].

distribution close to the wall. Interestingly enough, we find that aWDA shows good agreement with molecular simulations for the density distribution in the center of the pore in comparison with aFMT and iSAFT especially for the case of $H=3.02 \sigma$.

Even though the objective of this work is the study of the association contribution with classical DFT, in order to model real fluids it is required to take into account more interaction between the particles, such as dispersion forces, chain formation or even electrostatic forces. An interesting test example was carried out by Malheiro et al. [45]. They made a calculation of a system of associating hard spheres resembling water in a narrow slit with attractive walls, where besides the solid-fluid interaction, all dispersion forces were neglected. The solid-fluid interactions were modeled with the Steele's "10-4-3" potential [46], which parameters can be found in Ref. 45. For the test fluid, the particle diameter is $\sigma=3.033 \AA$, the association energy $\varepsilon_{A B} / k_{b}=1336.9$ $\mathrm{K}$, the associating volume $K=8.9369 \cdot 10^{-4} \AA^{3}$, and the solid-fluid interaction energy $\varepsilon_{s f} / k_{b}=91.717 \mathrm{~K}$, which is similar to the interaction energy between carbon and water [45]. The calculation is carried out for a pore of width $H=7 \AA$, with a system pressure of $P=0.5$ bar which correspond to a number bulk density of $8.517 \cdot 10^{-6}$ $\AA^{-3}$ at $T=425 \mathrm{~K}$. Fig. 16 shows the resulting density profiles calculated with the tree functionals in comparison with molecular simulations, where the agreement between theory and simulations is excellent. Moreover, the performance between the three functionals is virtually identical indicating that the system is dominated by the wallfluid interactions and not the association interactions. It is worth nothing that at these conditions we are working with adsorption of a water-like fluid in equilibrium with a bulk fluid in the vapor phase, which resembles a real case scenario where capillary condensation in porous media may occur [45]. 


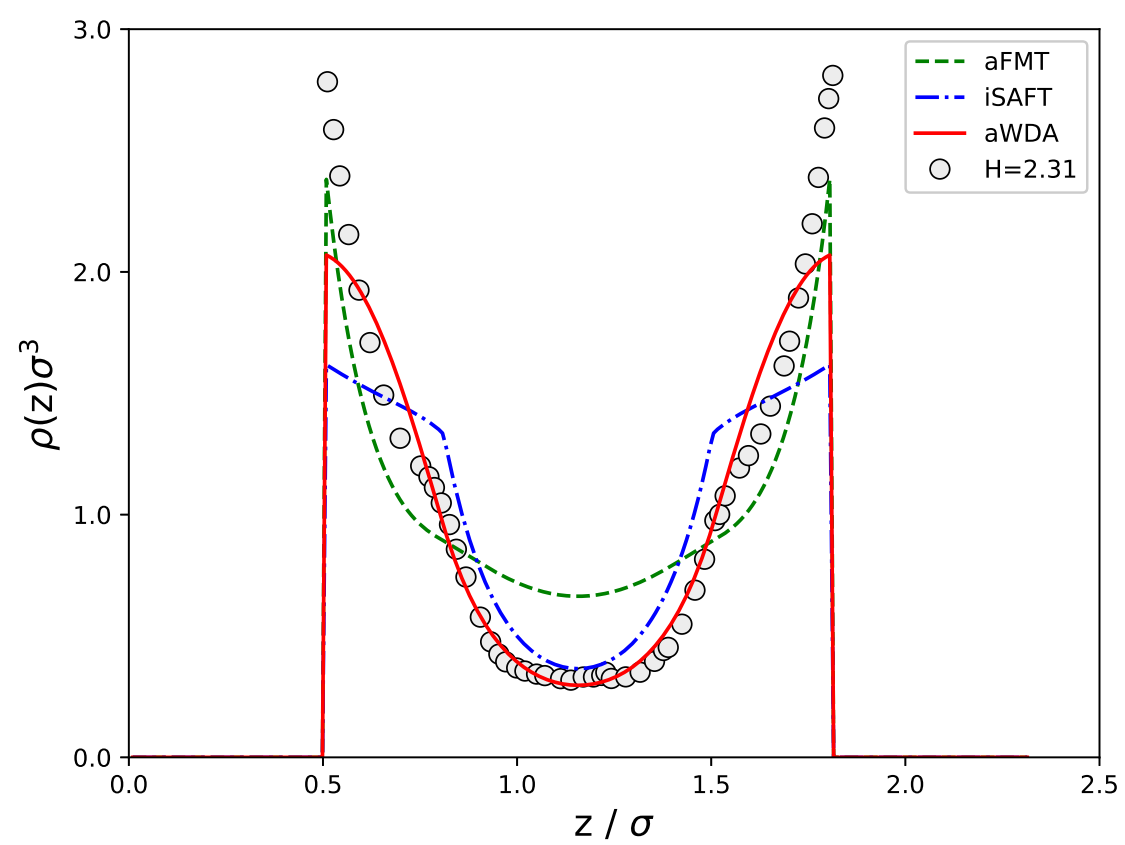

Figure 14. Same as Fig. 13, with $H=2.31 \sigma$. Symbols represent molecular simulations [42].

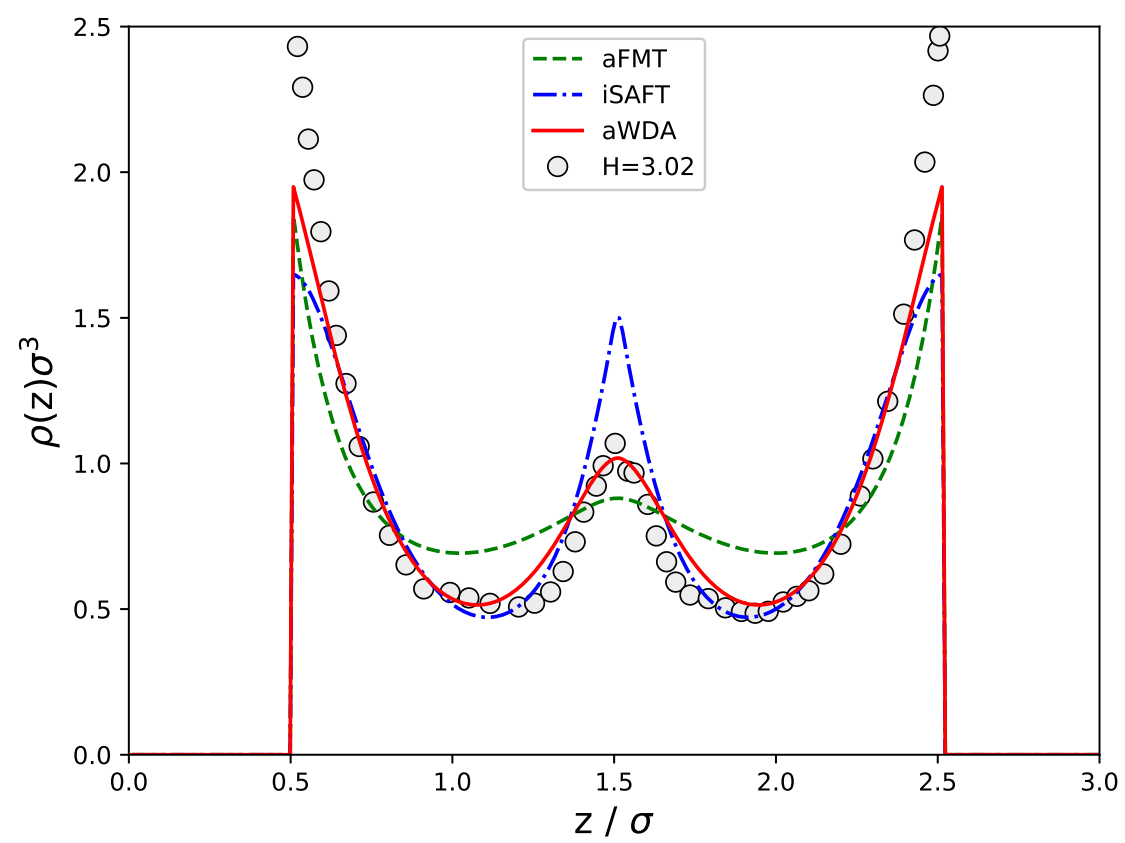

Figure 15. Same as Fig. 13, with $H=3.02 \sigma$. Molecular simulation results shown as symbols [42]. 


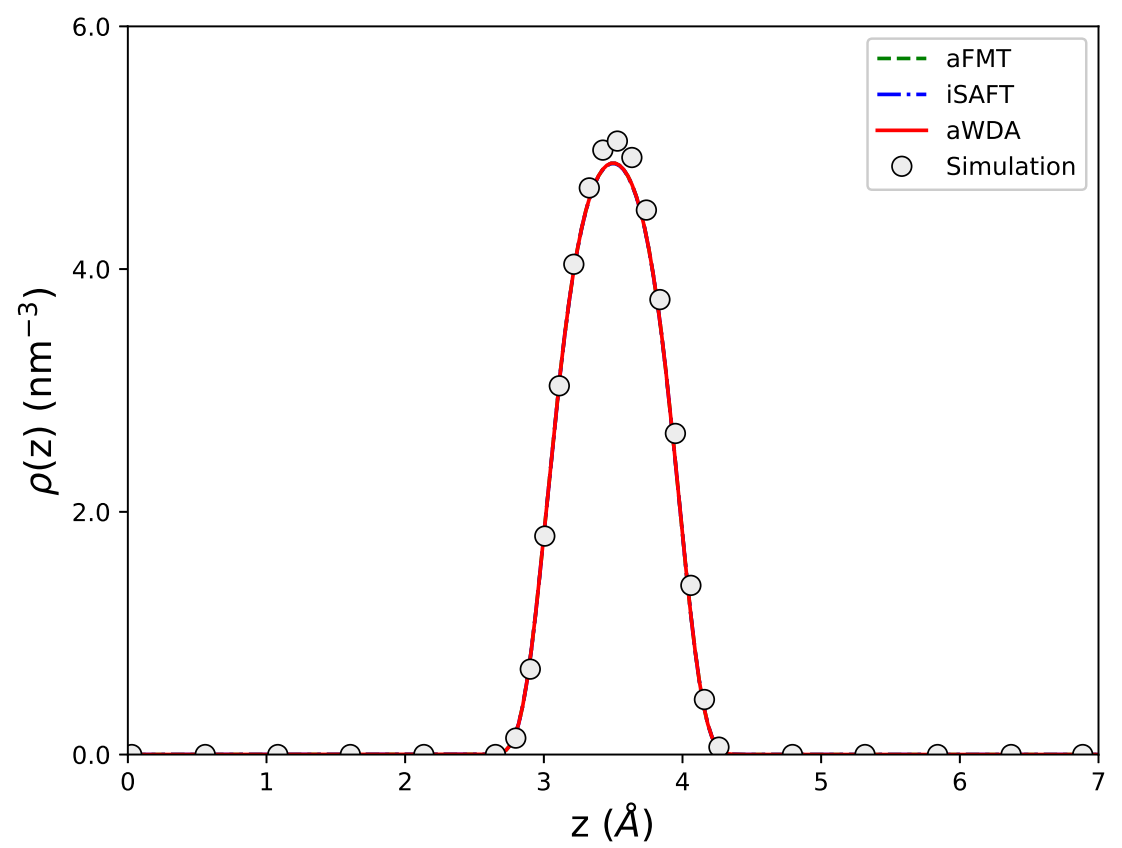

Figure 16. Density distribution of a hard sphere fluid with four association sites confined in a slit-pore with attractive walls. Lines are results obtained with classical DFT and three different association functionals and symbols are results from molecular simulations [45].

\subsection{Binary mixtures}

Following the steps of Segura et al. [32] and $\mathrm{Yu}$ and $\mathrm{Wu}[6]$, we tested our implementation with binary mixtures of an inert hard sphere fluid and the water-like model with four association sites close to a hard wall. Two different kinds of results are presented, one shows the ratio of the density of hard spheres to the density of associating spheres $\rho_{\mathrm{hs}}^{*}(z) / \rho_{\mathrm{ass}}^{*}(z)$. Secondly, we show the reduced density distribution $\rho_{\text {ass }}^{*}(z)$ of the associating hard spheres close to one of the walls of the slit.

Fig. 17 shows the results for the mixture of associating spheres with hard spheres for a relatively low density and moderate association energy. As shown in panel (a), there is a higher concentration of hard spheres close to the wall in comparison with the associating spheres. As observed in previous calculations, when the association is strong enough there is formation of clusters and due to excluded volume effects the hard spheres tend to accumulate close the wall excluding the larger cluster to the center of the slit. Additionally, as the wall is also inert the association of sites are restricted in the vicinity of the wall favoring depletion even more. All functionals perform well in comparison with molecular simulations.

In Fig. 18 we show the same binary system for a higher density and temperature. As opposed to Fig. 17, this system is clearly in conditions where the hard sphere contribution dominates over association. The behavior of the ratio of hard spheres to associating spheres is well captured by both aFMT and iSAFT, whereas aWDA predicts a smooth decrease in this profile as shown in (a). The ratio of hard spheres to associating spheres represents the relative difference between the density distributions of hard spheres to associating spheres. The fact that aWDA shows a smooth transitions indicates that it predicts similar trends for both density distributions, which implies that it fails to capture the effect that the mixing of both fluids have in the distribution 


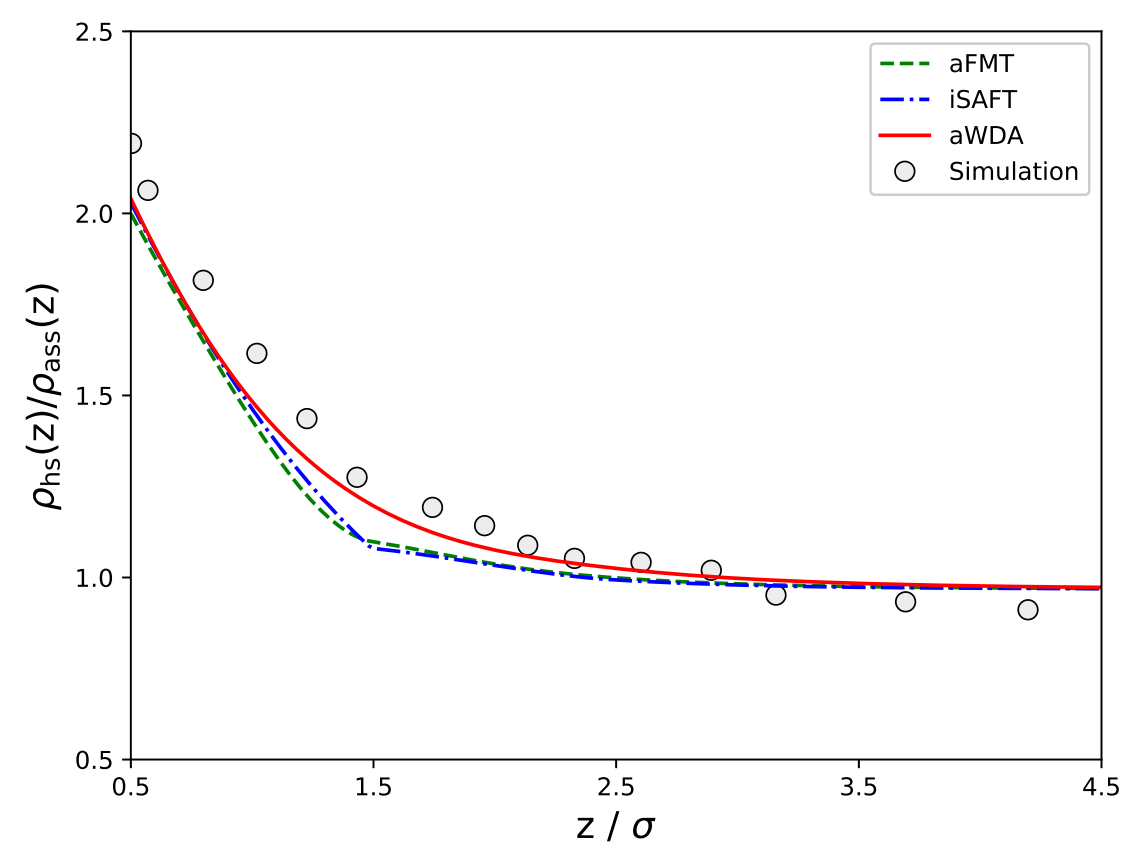

(a)

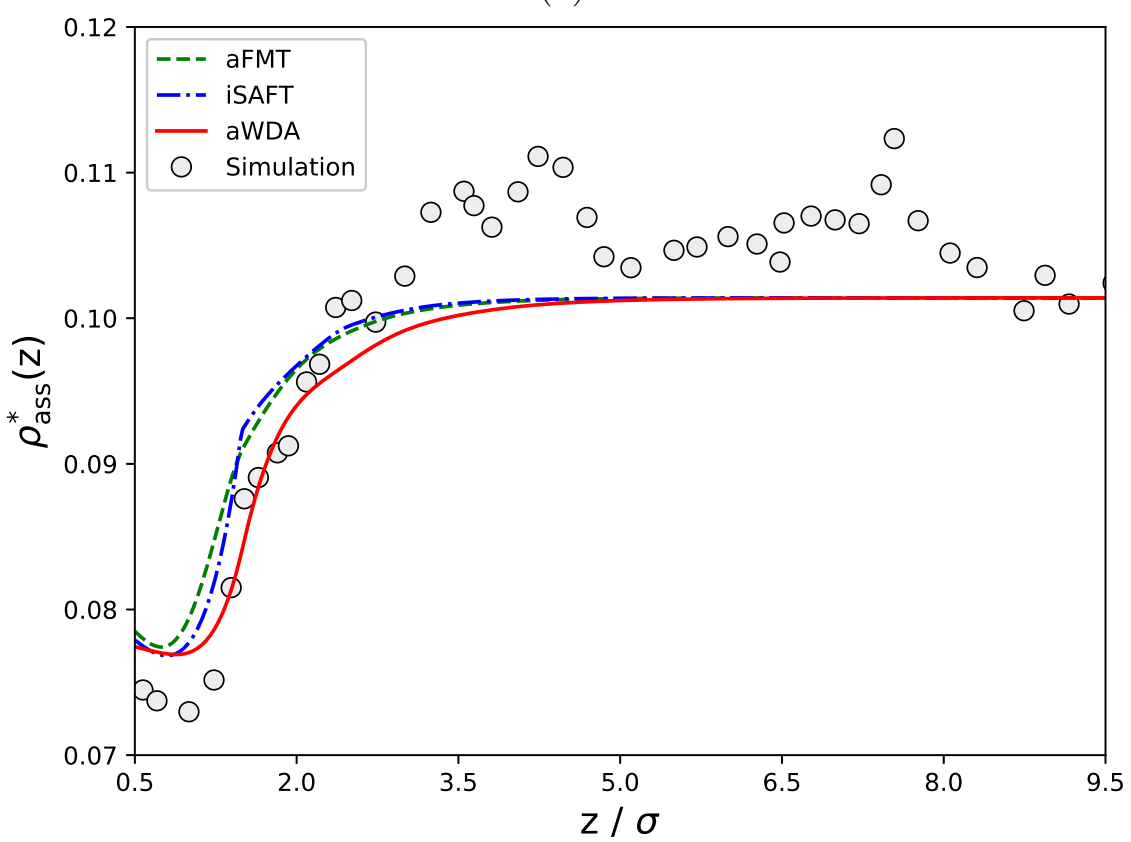

(b)

Figure 17. Results for a mixture of a fluid with four associating sites and hard spheres near a hard wall at $\rho_{\mathrm{b}}^{*}=0.1996,1 / T^{*}=7.0$, with a fraction of the associating fluid of $x_{a}=0.5080$. (a) Density ratio of hard spheres to associating spheres, and (b) shows the reduced density of associating spheres. Monte Carlo simulation results shown as symbols [32]. 
of particles close to the wall in comparison to the other functionals. However, the distribution of hard spheres in the mixture predicted using the three functionals is almost identical as exemplified in Fig. 19.

For the case on panel (b), there is very good agreement with simulation results for all functionals showing the layering effect typical of volume exclusion predominance.

Finally, the fraction of monomers for the two cases of binary mixtures are plotted in Fig. 20. Results obtained with the three classical DFT functionals agree perfectly with simulation results, but simulation shows oscillations further from the wall where classical DFT predicts a value corresponding to bulk conditions.

\section{Conclusion}

We proposed a new association density functional based in the bulk form of Wertheim's perturbation theory together with the weighted density approximation (aWDA). We paired our functional with the modified fundamental measure theory in order to account for volume exclusion effects and association of particles. The performance of our implementation in calculating the equilibrium density profile of fluids with different association schemes showed excellent agreement with results obtained with Monte Carlo simulations of the same systems at the same conditions [30-32, 39].

Although it is not the first time a WDA approach has been proposed for the association functional [10,30-32], previous implementations have not shown the degree of performance found by the most commonly used functionals of $\mathrm{Yu}$ and $\mathrm{Wu}$ (aFMT) [6], and Bymaster and Chapman (iSAFT) [10]. Reasons for this may be attributed to the definition of the weighting function in the calculation of the weighted density. Recent works have used a similar weighted density approach as the one we followed here with other free energy densities of bulk fluids $[25,47,48]$ where the range of weighting is controlled by a parameter that can be later fitted to the experimental data of interest and treated as a global parameter [26, 49].

Moreover, we tested the performance of the new association aWDA functional in comparison with aFMT and iSAFT. As we only took into account volume exclusion effects through modified FMT and association of particles, we could observe the individual effect that a given association functional has in relation with the competition about how these two contributions influence the behavior of the density profile in confined associating fluids. Based on the results presented here, we confirmed that the three association functionals are able to predict the behavior of associating fluids with different number of bonding sites. In general, iSAFT shows slightly better agreement with simulations for confined fluids with one bonding site at low temperatures and densities. However, at higher densities and temperatures, where the associating effects are not very pronounced all three functionals show equivalent performance. For the cases of two and four bonding sites studied here, iSAFT and aWDA were found to be more in agreement with simulations for most of the cases. For binary mixtures of a hard sphere fluid with an associating fluid with four bonding sites all three functionals have excellent agreement for the density distribution of associating spheres and some discrepancies in the ratio of hard spheres to associating spheres. For narrow slits, we found that classical DFT with the three associationg functionals is able to describe the layering transitions of the density distribution of associating fluids with four active sites when compared to molecular simulations with mild deviations. Finally, it is important to note that the computational time required by iSAFT is far larger that for aFMT and aWDA. This is because the calculation of $\chi_{A_{i}}(z)$ for aFMT and aWDA 


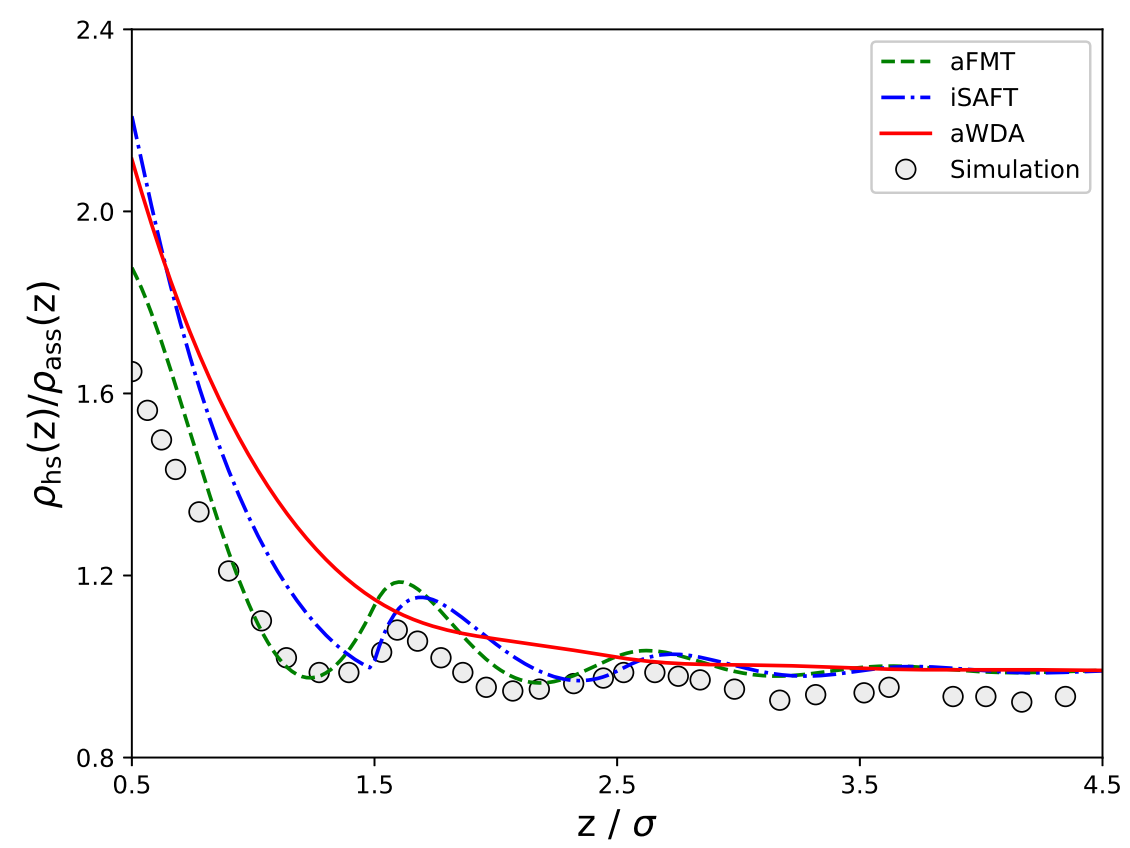

(a)

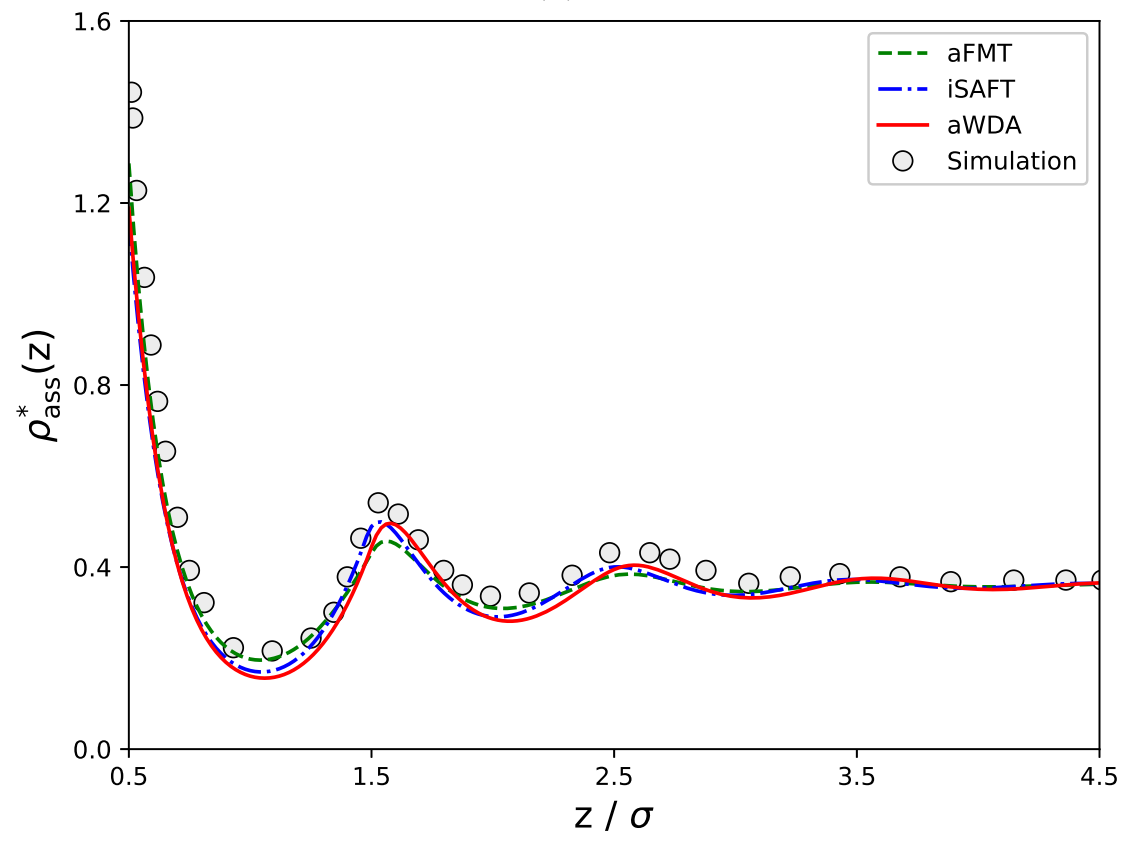

(b)

Figure 18. Results for a mixture of a inert fluid with four associating sites and hard spheres near a hard wall at $\rho_{\mathrm{b}}^{*}=0.7173,1 / T^{*}=5.0$, with a fraction of the associating fluid of $x_{a}=0.5024$. (a) Density ratio of hard spheres to associating spheres, and (b) shows the reduced density of associating spheres. Monte Carlo simulation results shown as symbols [32]. 


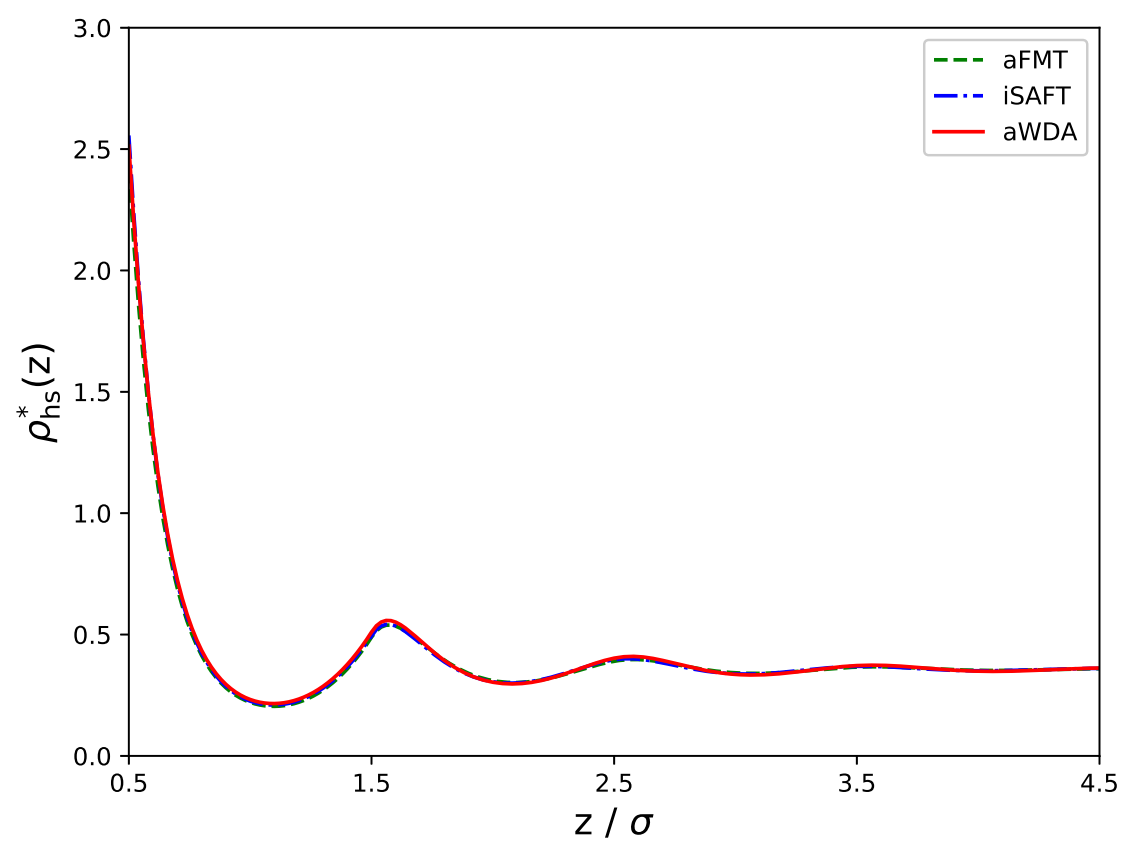

Figure 19. Density distribution of hard spheres in the mixture with associating particles at $\rho_{\mathrm{b}}^{*}=0.7173$, $1 / T^{*}=5.0$, with a fraction of the associating fluid of $x_{a}=0.5024$.

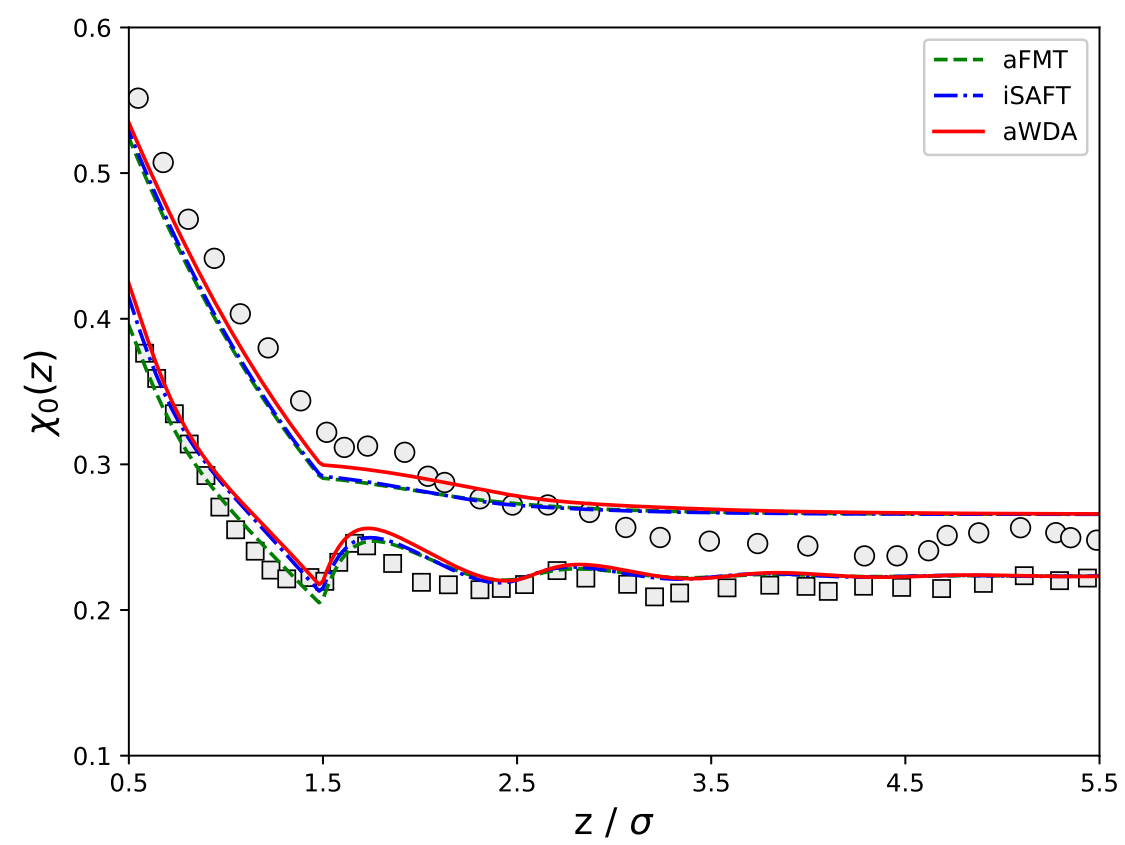

Figure 20. Comparison of the monomer fraction of the fluid with four association sites calculated with three different functionals, symbols show Monte Carlo simulation results [30]. In the top at $\rho_{\mathrm{b}}^{*}=0.1996,1 / T^{*}=7.0$ with $x_{a}=0.5080$, and bottom $\rho_{\mathrm{b}}^{*}=0.7173,1 / T^{*}=5.0$ with $x_{a}=0.5024$. 
is a local operation, whereas for iSAFT the neighboring values of $\chi_{A_{i}}$ around $z$ are required in the integral. As example, for results shown in Fig. 17 the calculation time required by aFMT and aWDA is around $24-31$ seconds respectively, whereas for iSAFT we obtained about 36 minutes with a standard Piccard iteration procedure. For this comparison we carried out the calculation in the same computer and with the same parameters values used in the iterative process. For this reason we conclude that aWDA can become a strong candidate for studying inhomogeneous associating fluids by having an excellent agreement with simulation results and only requires a moderate computation time making it fit for both research and industry applications.

\section{References}

[1] J.P. Hansen and I.R. McDonald, Theory of Simple Liquids with Applications to Soft Matter, 4th ed. (Academic Press, Oxford, 2013).

[2] J. Wu, AIChE J. 52 (3), 1169-1193 (2006).

[3] R. Roth, R. Evans, A. Lang and G. Kahl, J. Phys. Condens. Matter 14 (02), 12063-12078 (2002).

[4] R. Roth, J. Phys. Condens. Matter 22, 63102-18 (2010).

[5] Y.X. Yu and J. Wu, J. Chem. Phys. 117 (22), 10156-10164 (2002).

[6] Y.X. Yu and J. Wu, J. Chem. Phys. 116 (16), 7094-7103 (2002).

[7] Y.X. Yu and J. Wu, J. Chem. Phys. 117 (5), 2368-2376 (2002).

[8] S. Tripathi and W.G. Chapman, J. Chem. Phys. 122 (9), 1-11 (2005).

[9] Y. Tang and B.C.Y. Lu, Fluid Phase Equilib. 190, 149-158 (2001).

[10] A. Bymaster and W.G. Chapman, J. Phys. Chem. B 114 (38), 12298-12307 (2010).

[11] J. Wu and Z. Li, Annu. Rev. Phys. Chem. 58 (1), 85-112 (2007).

[12] C.P. Emborsky, Z. Feng, K.R. Cox and W.G. Chapman, Fluid Phase Equilib. 306 (1), 15-30 (2011)

[13] M.S. Wertheim, J. Stat. Phys. 35 (1-2), 19-34 (1984).

[14] M.S. Wertheim, J. Stat. Phys. 35 (1-2), 35-47 (1984).

[15] M.S. Wertheim, J. Stat. Phys. 42 (3-4), 459-476 (1986).

[16] M.S. Wertheim, J. Stat. Phys. 42 (3-4), 477-492 (1986).

[17] G. Jackson, W.G. Chapman and K.E. Gubbins, Int. J. Thermophys. 9 (5), 769-779 (1988).

[18] W.G. Chapman, G. Jackson and K.E. Gubbins, Mol. Phys. 65 (5), 1057-1079 (1988).

[19] W.G. Chapman, K.E. Gubbins, G. Jackson and M. Radosz, Ind. Eng. Chem. Res. 29 (8), 1709-1721 (1990).

[20] J. Gross and G. Sadowski, Ind. Eng. Chem. Res. 40 (4), 1244-1260 (2001).

[21] J. Gross and G. Sadowski, Ind. Eng. Chem. Res. 41 (22), 5510-5515 (2002).

[22] A. Gil-Villegas, A. Galindo, P.J. Whitehead, S.J. Mills, G. Jackson and A.N. Burgess, J. Chem. Phys. 106 (10), 4168-4186 (1997).

[23] Y. Rosenfeld, Phys. Rev. Lett. 63 (9), 980-983 (1989).

[24] G.J. Gloor, F.J. Blas, E.M. Del Río, E. De Miguel and G. Jackson, Fluid Phase Equilib. 194-197, 521-530 (2002).

[25] G. Shen, X. Ji and X. Lu, J. Chem. Phys. 138, 224706 (2013).

[26] E. Sauer and J. Gross, Ind. Eng. Chem. Res. 56, 4119-4135 (2017).

[27] D. Fu and J. Wu, Mol. Phys. 102 (13), 1479-1488 (2004).

[28] D. Ghonasgi and W.G. Chapman, Mol. Phys. 79 (2), 291-311 (1993).

[29] C. J. Segura and W. G. Chapman, Mol. Phys. 86 (3), 415-442 (1995).

[30] C.J. Segura, W.G. Chapman and K. Shukla, Mol. Phys. 90 (5), 759-771 (1997).

[31] C.J. Segura, E.V. Vakarin, W.G. Chapman and M.F. Holovko, J. Chem. Phys. 108 (12), 4837-4848 (1998).

[32] C.J. Segura, J. Zhang and W.G. Chapman, Mol. Phys. 99 (1), 1-12 (2001).

[33] R. Evans, in Fundam. Inhomogeneous Fluids, edited by Douglas Henderson, Chap. Third 
(Marcel Dekker, Inc., New York, 1992), pp. 85-176.

[34] J. Hughes, E.J. Krebs and D. Roundy, J. Chem. Phys. 138 (2) (2013).

[35] V.M. Trejos, S. Sokolowski and O. Pizio, J. Chem. Phys. 149 (13) (2018).

[36] V.M. Trejos, S. Sokolowski and O. Pizio, Mol. Phys. 8976 (May) (2019).

[37] P. Tarazona and R. Evans, Mol. Phys. 52, 847-857 (1984).

[38] W.A. Curtin and N.W. Ashcroft, Phys. Rev. A 32 (5), 2909-2919 (1985).

[39] A. Patrykiejew, S. Sokołowski and D. Henderson, Mol. Phys. 95 (2), 211-218 (1998).

[40] S.H. Huang and M. Radosz, Ind. Eng. Chem. Res. 29 (11), 2284-2294 (1990).

[41] S.H. Huang and M. Radosz, Ind. Eng. Chem. Res. 30 (8), 1994-2005 (1991).

[42] J. Galle and H.L. Voertler, Surf. Sci. 421, 33-43 (1999).

[43] H.L. Voertler and M. Kettler, Chem. Phys. Lett. 266 (3-4), 368-374 (1997).

[44] R.P. Brent, Algorithms for Minimization Without Derivatives (NJ: Prentice-Hall, Englewood Cliffs, 1973), pp. Ch. 3-4.

[45] C. Malheiro, B. Mendiboure, J.M. Míguez, M.M. Piñeiro and C. Miqueu, J. Phys. Chem. C 118 (43), 24905-24914 (2014).

[46] W.A. Steele, Surf. Sci. 36, 317-352 (1973).

[47] Z. Ye, J. Cai, H. Liu and Y. Hu, J. Chem. Phys. 123, 194902 (2005).

[48] G. Shen, X. Ji, S. Öberg and X. Lu, J. Chem. Phys. 139, 194705 (2013).

[49] E.L. Camacho Vergara, G.M. Kontogeorgis and X. Liang, Ind. Eng. Chem. Res. 58, 56505664 (2019). 